Meta

Journal des traducteurs

Translators' Journal

\title{
Comparaison des traductions japonaise et chinoise de Paul Morand pendant les années 1920 et 1930
}

\section{Sabrina Choi-Kit Yeung}

Volume 61, numéro 2, août 2016

URI : https://id.erudit.org/iderudit/1037764ar

DOI : https://doi.org/10.7202/1037764ar

Aller au sommaire du numéro

\section{Éditeur(s)}

Les Presses de l’Université de Montréal

ISSN

0026-0452 (imprimé)

1492-1421 (numérique)

Découvrir la revue

Citer cet article

Yeung, S. C. (2016). Comparaison des traductions japonaise et chinoise de Paul Morand pendant les années 1920 et 1930. Meta, 61(2), 369-395.

https://doi.org/10.7202/1037764ar
Résumé de l'article

Pour comprendre l'émergence des mouvements modernistes dans les littératures japonaise et chinoise, au cours des années 1920 et 1930, « le modernisme ", « l'École des sensations nouvelles " et " Paul Morand " sont trois jalons incontournables. Nous proposons dans cet article une comparaison des mouvements modernistes japonais et chinois à partir de la traduction, en nous concentrant sur deux nouvelles de Paul Morand : La nuit romaine et La nuit des six-jours qui ont été traduites en japonais et en chinois au cours de cette période. Grâce à l'étude comparée des traductions, nous tentons de répondre aux questions suivantes : comment le statut culturel des traducteurs a-t-il influencé leurs stratégies de traduction ? En quoi les stratégies de traduction japonaise et chinoise ont-elles été vectrices d'une certaine forme de modernité ? Comment les choix de traduction ont-ils dévoilé les multiples facettes de la modernité des deux pays ? 


\title{
Comparaison des traductions japonaise et chinoise de Paul Morand pendant les années 1920 et 1930
}

\author{
SABRINA CHOI-KIT YEUNG \\ Université Paris 4 - Paris-Sorbonne, Paris, France \\ walawalakit@hotmail.com
}

\begin{abstract}
RÉSUMÉ
Pour comprendre l'émergence des mouvements modernistes dans les littératures japonaise et chinoise, au cours des années 1920 et 1930, «le modernisme», «l'École des sensations nouvelles» et «Paul Morand» sont trois jalons incontournables. Nous proposons dans cet article une comparaison des mouvements modernistes japonais et chinois à partir de la traduction, en nous concentrant sur deux nouvelles de Paul Morand: La nuit romaine et La nuit des six-jours qui ont été traduites en japonais et en chinois au cours de cette période. Grâce à l'étude comparée des traductions, nous tentons de répondre aux questions suivantes: comment le statut culturel des traducteurs a-t-il influencé leurs stratégies de traduction? En quoi les stratégies de traduction japonaise et chinoise ont-elles été vectrices d'une certaine forme de modernité? Comment les choix de traduction ont-ils dévoilé les multiples facettes de la modernité des deux pays?
\end{abstract}

\begin{abstract}
In order to understand the emergence of modernist movements in Japanese and Chinese modern literature, "modernism," "Neo-Perceptionist School" and "Paul Morand" are three keywords closely intertwined. This article proposes to compare the Japanese and Chinese modernist movements from a more original step - the translation, by focusing on Paul Morand's two short novels: The Roman Night and The Night of Six-days, which were simultaneously translated into Japanese and Chinese during the modernist movement in 1920s and 1930s. Through the comparison, we try to answer the following questions: how did the cultural roles of translators influence their translation strategies? In what way have the Japanese and Chinese translation strategies shown modernity? How did the differences of Japanese and Chinese translation strategies reveal different facets of modernity in these two countries?
\end{abstract}

\section{MOTS-CLÉS/KEYWORDS}

modernisme japonais, modernisme chinois, école des sensations nouvelles, traduction japanese modernism, chinese modernism, neo-perceptionist school, translation

\section{Introduction}

Pour comprendre l'émergence des mouvements modernistes dans les littératures japonaise et chinoise des années 1920 et 1930, «le modernisme», «l’École des sensations nouvelles» et «Paul Morand» sont trois jalons incontournables. Comment «cette relation triangulaire» s'est-elle formée?

En ce qui concerne le Japon, presque tous les critiques littéraires japonais de la fin des années 1920 jusqu'à aujourd'hui considèrent «l'École des sensations nouvelles» 
(新感覚派 $)^{1}$ comme un concept clé pour définir le mouvement moderniste japonais, comme l'a souligné Chiba Sen'ichi (1930-) (Chiba 1978: 150):

Analyser les débats autour de l'École des sensations nouvelles, discuter son importance dans l'histoire littéraire, peu importe de quel point de vue, c'est une prémisse indispensable pour clarifier les singularités et les frontières de la littérature moderniste du Japon [...]. (Notre traduction) ${ }^{2}$

Qu'en est-il du rôle de Paul Morand (1888-1976) ? Quel est son lien avec «l'École des sensations nouvelles» et «le modernisme» ? Paul Morand a pris part au mouvement moderniste japonais des années 1920 à travers l'École des sensations nouvelles. La traduction japonaise de son œuvre représentative Ouvert la nuit (1922) ${ }^{3}$ entreprise par Horiguchi Daigaku (1892-1981), réimprimée douze fois en deux ans (1924-1926), lui a assuré une grande célébrité au Japon au milieu des années 1920. Par la suite, Chiba Kameo (1878-1935), l'un des meilleurs critiques littéraires de l'époque, a remarqué des similitudes entre le style littéraire de Morand et celui des écrivains de l'École des sensations nouvelles (Chiba 1924, 194)4. À travers cette proximité stylistique, Morand a été associé au mouvement moderniste japonais, car on pensait que son style littéraire était un élément essentiel à la pratique moderniste de l'École des sensations nouvelles japonaise.

Dans le cas de la Chine, la rencontre de ces trois parties - "le modernisme», «l'École des sensations nouvelles» et «Paul Morand»- a été rendue possible par un jeune écrivain moderniste chinois, Liu Na'ou (1905-1940). Au moment où l'École des sensations nouvelles occupait la position de «littérature pour la jeune génération» dans le champ littéraire du milieu des années 1920 au Japon, la vénération pour Morand a également attiré l'attention de Liu qui poursuivait ses études secondaire et universitaire au Japon (1920-1926). Lors de son séjour, il a partagé l'engouement de la jeunesse japonaise pour les œuvres de Morand par le biais des traductions japonaises ${ }^{5}$. À son retour en Chine en 1926, Liu est devenu à la fois un acteur décisif de l'introduction de Morand en Chine, de la fondation de l'École des sensations nouvelles chinoise et du mouvement moderniste chinois des années 1930. Grâce au concours de Liu, Morand a laissé une image d'avant-gardiste dans l'histoire littéraire moderne chinoise, ce que l'écrivain français n'avait probablement pas anticipé. Dans ce contexte historique, deux Écoles des sensations nouvelles, le modernisme et Paul Morand, se sont croisés sur les scènes des mouvements modernistes japonais et chinois pendant les années 1920 et 1930.

On pourrait facilement établir un lien entre un écrivain français (Paul Morand), un courant littéraire issu de l'Europe (le modernisme) et deux champs littéraires asiatiques (le Japon et la Chine des années 1920 et 1930) dans la catégorie des études de la réception, au sein desquelles on met en évidence le décalage entre «le textesource» et "la culture-cible», en analysant comment les différences culturelles ont contribué à ce décalage, ou comment les écrivains asiatiques ont tourné les formes européennes à leurs propres fins. Mais les études de traduction révèlent deux défauts inhérents à ce genre de méthodologie.

Premièrement, avant qu'il y ait un décalage entre «le texte-source» et «la culturecible», la traduction elle-même fait déjà subir des distorsions au texte original. Ce fut le cas lors de l'introduction des œuvres de Morand au Japon et en Chine pendant les années 1920 et 1930, parce que les traducteurs japonais et chinois étaient investis de 
plusieurs rôles culturels tels que poète, traducteur, chef de courant littéraire, intellectuel, écrivain moderniste. Leurs rôles culturels, notamment ceux de poète et d'intellectuel s'engageant à disséminer la culture moderniste, ont beaucoup influencé leurs stratégies de traduction. Dès lors, pour se donner une chance de comprendre les enjeux véritables d'un texte diffusé à l'étranger, il faut analyser la position du traducteur qui participe au processus de consécration de l'œuvre.

Deuxièmement, dans la perspective de la réception de l'œuvre, le texte traduit serait important pour la transformation de la culture-cible parce qu'il est porteur d'idées étrangères, il rend le texte-source compréhensible pour le lecteur local, c'està-dire qu'il est un canal ouvert à travers lequel les influences étrangères peuvent pénétrer dans la culture locale, la contester, même contribuer à la subvertir pour achever une transformation culturelle. Par exemple, au début de l'ère Meiji (18681912), les romans de politiciens comme ceux d'Edward Bulwer-Lytton (1803-1873) ${ }^{6}$ et de Benjamin Disraeli (1804-1881) ${ }^{7}$, ainsi que les œuvres de science-fiction de Jules Verne (1828-1905) ${ }^{8}$ ont été traduits ou adaptés en japonais dans le but d'aider le Japon à devenir plus puissant et de le pousser vers le chemin de la modernité, en transmettant les modèles occidentaux de gouvernement et les pensées politiques occidentales. Cependant, avant de se poser la question des relations entre traduction et transformation de la culture - dans le cas du Japon, cela sous-entend achever la modernité par la traduction - il faut d'abord se demander quel genre de modernité est la traduction elle-même. En effet, à force de se concentrer sur ses relations avec la modernité, on risque d'oublier que la traduction est elle aussi une modernité et donc partie intégrante de la modernité. À titre d'exemple, dans la traduction chinoise des œuvres de Morand, le traducteur Dai Wangshu (1905-1950) a adopté la stratégie de la traduction littérale afin de préserver la syntaxe, la grammaire et le vocabulaire français. Sa traduction a souvent enfreint les règles de la syntaxe chinoise ou été confrontée au problème de la traduction raide (硬譯), une manière de traduction mot-à-mot qui aboutit à un texte parfois incompréhensible ${ }^{9}$. Pour Dai, cette manière de traduction pourrait enrichir ou compléter la langue chinoise moderne en y introduisant des éléments de la langue occidentale. En réalité, cette stratégie d'« européaniser» la langue chinoise était censée être favorable au développement du chinois vernaculaire, une étape décisive pour se débarrasser de la tradition. Donc, avant même d'analyser le contenu des textes traduits, nous nous rendons compte que le choix de la traduction littérale par Dai Wangshu manifeste déjà une sorte de modernité chinoise. Nous discuterons davantage de ce problème dans la partie consacrée à «la stratégie de la traduction chinoise». En somme, les études de traductologie nous montrent que la traduction n'est pas seulement porteuse des idées modernes, elle est aussi une facette de la modernité, surtout quand plus d'attention est accordée aux questions linguistiques.

Ainsi, même si plusieurs recherches ont été effectuées sur les modernismes japonais et chinois et sur les Écoles des sensations nouvelles japonaise et chinoise ${ }^{10}$, nous proposons dans cet article une comparaison des mouvements modernistes japonais et chinois à partir de la traduction, en nous concentrant sur deux nouvelles provenant d'Ouvert la nuit de Paul Morand: La nuit romaine (1922) et La nuit des six-jours (1922) qui ont été traduites en japonais et en chinois pendant les courants modernistes japonais et chinois des années 1920 et 1930. À travers l'étude comparée des traductions, nous tenterons d'aboutir à trois constatations: tout d'abord, le statut 
social des traducteurs, considérés comme le poète et les têtes de file du courant littéraire moderniste, a influencé leurs stratégies de traduction; ensuite, les stratégies de traduction japonaise et chinoise ont été vectrices d'une certaine forme de modernité; enfin, les différences en matière de choix de traduction ont dévoilé les multiples facettes de la modernité des deux pays.

\section{Traduction japonaise des œuvres de Paul Morand}

\subsection{Horiguchi Daigaku: poète et traducteur}

La publication, en novembre 1922, de la moitié de 北欧の夜 [La nuit nordique, 1922] (Tsuchiya 2006, 99) ${ }^{11}$ dans la revue littéraire 明星 [Étoile du matin] a inauguré la vague de traduction en langue japonaise des œuvres de Morand. Horiguchi Daigaku a accompli la plus grande partie de cette tâche. Il a grandement contribué à l'établissement de la réputation de Morand au Japon. Une liste des traductions qu'il a entreprise des œuvres de Morand pendant les années 1920 suit (Ōmura 2011: 85):

TABLEAU 1

La traduction japonaise des œuvres de Paul Morand entreprise par Horiguchi Daigaku dans les années 1920

\begin{tabular}{|c|c|}
\hline Année & Titre \\
\hline $\begin{array}{l}1922 \\
\text { (Taishō 11) }\end{array}$ & $\begin{array}{l}\text { Novembre } \\
\text { 北欧の夜 [La nuit nordique]. In: 明星 [Étoile du matin], vol. 2, nº } 6 \\
\text { (une partie de «La nuit nordique») }\end{array}$ \\
\hline $\begin{array}{l}1924 \\
\text { (Taishō 13) }\end{array}$ & $\begin{array}{l}\text { Juillet } 15 \\
\text { 夜開 }<\text { [Ouvert la nuit], Tokyo: Shinchōsha }\end{array}$ \\
\hline $\begin{array}{l}1925 \\
\text { (Taishō 14) }\end{array}$ & 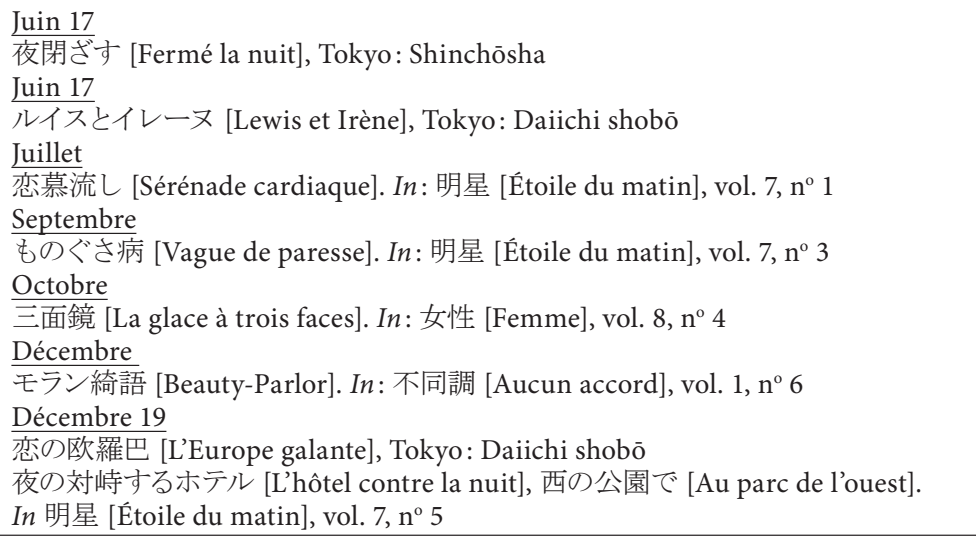 \\
\hline $\begin{array}{l}1926 \\
\text { (Taishō 15, } \\
\text { Shōwa 1) }\end{array}$ & $\begin{array}{l}\text { Janvier } \\
\text { «Clarisse». In: 明星 [Étoile du matin], vol. 8, n }{ }^{\circ} 1 \\
\text { Avril } \\
\text { 公衆浴場 [Bains publics], 敬礼 [Révérence]. In 明星 [Étoile du matin], vol. 8, n } \mathrm{n}^{\circ} 3 \\
\text { «Business». In 文章往来 [Trafic littéraire], vol. } 1, \mathrm{n}^{\circ} 4\end{array}$ \\
\hline $\begin{array}{l}1927 \\
\text { (Shōwa 2) }\end{array}$ & $\begin{array}{l}\text { Octobre } \\
\text { «Delphine».In: 改造 [Reconstruction], vol. 9, n } \mathrm{n}^{\circ} 10\end{array}$ \\
\hline $\begin{array}{l}1928 \\
\text { (Shōwa 3) }\end{array}$ & $\frac{\text { Janvier } 5}{\text { Tendres Stocks, Tokyo : Daiichi shobō }}$ \\
\hline
\end{tabular}


Horiguchi est un traducteur réputé de la poésie française. Son recueil fameux de traduction de poèmes français, 月下の一群 [Un troupeau au clair de lune] (1925), apporte au monde littéraire japonais un excellent échantillon de la poésie française, contenant 340 poèmes de soixante-six auteurs, allant de Baudelaire, Verlaine ou Mallarmé à Guillaume Apollinaire et Jean Cocteau. Cet ouvrage a eu une forte influence sur le monde littéraire japonais à partir de la fin des années 1920. Ses connaissances en langue et littérature françaises ont été un élément essentiel pour gagner la confiance des lecteurs japonais. Son long séjour à l'étranger ${ }^{12}$, sa belle-mère belge, sa connaissance personnelle des artistes européens ${ }^{13}$, sa rencontre avec Morand à Paris ont également été des facteurs déterminants. Donc, les lecteurs ne remettaient pas en doute la fidélité de sa traduction, comme Ikuta Chōkō (1881-1936) l'a noté: "Je n'ai aucun doute quant à la capacité de traduction de M. Horiguchi ${ }^{14}$.»

Avant d'être traducteur, Horiguchi s'est fait connaître dans le monde littéraire par ses poèmes. Son premier recueil de poèmes 月光とピエロ [Le clair de lune et Pierrot] (1919) ${ }^{15}$ révèle l'influence du symbolisme sur sa pensée. Progressivement, il a évolué d'une inclinaison à la mélancolie dans [Le clair de lune et Pierrot] à un lyrisme subtil (Amoia et Knapp 2002: 184-185). Le processus a abouti à son quatrième recueil, 砂の枕 [L'oreiller de sable] (1926), dans lequel les thèmes ont été traités avec ironie et humour et organisés à partir d'une langue rythmée et concise. En vérité, l'une des contributions principales d'Horiguchi à la poésie japonaise est la création d'une forme de lyrisme sensuel soutenu par des expressions concrètes, éloignée du sentimentalisme ou de la décence conventionnelle. Cette inclinaison poétique a influencé ses traductions d'œuvres littéraires françaises, qui vont souvent au-delà de la simple transposition linguistique.

En tant que poète, Horiguchi a fréquemment mis l'accent sur l'intensité sensorielle et poétique des nouvelles de Morand. Au fil de sa réception de Morand, il a multiplié les éloges du style sensoriel de l'écriture morandienne, comme en témoigne sa célèbre préface d'Ouvert la nuit:

Le style de Paul Morand nous étonne. Pourquoi La réponse est très simple. Il est un styliste doté d'une grande sensibilité et d'un sens aigu de l'observation qui a créé de nouvelles connexions entre les objets que les moyens d'écriture précédents n'auraient jamais tenté de rassembler. La connexion entre les objets dans l'écriture ordinaire s'effectue suivant une «logique de la raison». Cependant, chez Morand, cette «logique de la raison» cède la place à une «logique de la sensation». (Horiguchi 1986: 220, notre traduction $)^{16}$

Horiguchi a pensé que l'insistance de Morand sur le style a donné à ses nouvelles une intense sensibilité et a ainsi favorisé l'émergence d'un monde de sensations [感覚の世界] (Horiguchi 1986: 221). Peu à peu, Horiguchi s'est intéressé à la capacité de critique de Morand sur la société de l'entre-deux-guerres. Il a écrit plusieurs essais consacrés à l'écrivain français après la Seconde Guerre mondiale. En 1958, Horiguchi a traduit L'Homme pressé (1941) ${ }^{17}$, qui est la dernière traduction japonaise de l'œuvre de Morand. Dans l'ensemble, aux yeux d'Horiguchi, Morand était avant tout un nouvelliste moderniste qui a décrit la vie moderne et cosmopolite en employant des métaphores et des images originales. 


\subsection{La manipulation de l'image}

S’il y a une légère évolution au fil de la réception de Morand par Horiguchi, la plupart des critiques littéraires des années 1920 et des membres de l'École des sensations nouvelles se sont focalisés sur le style sensoriel et métaphorique chez Morand ${ }^{18}$. Les phrases traduites littéralement comme celles-ci: «l'autre était vêtue de muscles maigres, veines en écharpes, ligatures de bracelets, ombres d'os et un visage animal sacré» (Morand 1992: 150) [もう一人の方の女は、瘦せた筋肉を着て、靜脈を襟卷に して、腕環の關節を持つた、骨の影の目立つ、神々しい獸物のやうな顏をした女であ る] (Horiguchi 1986: 294) et «un dahlia entra dans ma bouche ouverte jusqu'au gosier. Bataille de fleurs. Un jardin passait dans l'air» (Morand 1992: 150) [私の開 いた口の中へ、咽喉の奥までダリヤの花が一輪とびこんだ。花合戰。花園が空中に で浮ん消えた] (Horiguchi 1986: 294), ont été considérées comme des modèles au niveau stylistique pour l'École des sensations nouvelles dans leur pratique de l'écriture moderniste.

Morand a laissé au Japon l'image d'un nouvelliste porté sur le style et moderniste, d'une part, parce que, hormis L'Homme pressé, Horiguchi n'a traduit que les premières œuvres de Morand qui donnent encore au lecteur d'aujourd'hui l'impression d'avoir affaire à une prose très élaborée et constamment tirée vers le poème. D’autre part, les stratégies de traduction d'Horiguchi, comme la manipulation de l'image, ont contribué à accentuer l'originalité du style métaphorique de Morand. Par conséquent, dans cette partie, nous discuterons de la manipulation de l'image dans la traduction d'Horiguchi, puis nous nous poserons les questions suivantes: quel est le rapport entre le style métaphorique de Morand et le modernisme de la littérature japonaise dans le contexte littéraire des années 1920 ? Pourquoi son style est-il davantage considéré comme un élément essentiel à la pratique moderniste de l'École des sensations nouvelles?

La manipulation de l'image est ici axée sur la stratégie de l'ajout de descriptions aux images et aux métaphores et l'ajout de personnification du traducteur aux textes de Morand. Dans La nuit romaine, l'héroïne Isabelle se trouve dans une situation paradoxale: d'une part, elle est très émancipée grâce à la modernité de son époque, via son penchant pour un mode de vie hédoniste; d'autre part, elle n'est pas bien intégrée à son temps. Dans le premier paragraphe, le narrateur relate d'abord la rencontre entre le protagoniste et la mère d'Isabelle. Et à travers la conversation entre ceux-ci s'impose l'image d'Isabelle. Horiguchi a multiplié les passages descriptifs pour souligner l'image mondaine de la mère d'Isabelle, et un peu plus loin, dans le deuxième paragraphe, Horiguchi a ajouté d'autres descriptions afin de mettre l'accent sur l'inadaptation au monde réel d'Isabelle, ce qui la conduit à se suicider. L'ajout de traits supplémentaires à cette évocation de deux images féminines crée un contraste entre mère et fille. Au début du premier paragraphe, le narrateur conclut son observation de la mère d'Isabelle par une phrase: «C'était une petite femme excessivement conservée par le lait de concombres et l'égoïsme. Les rides du visage nouées derrière l'oreille, la poitrine ensemencée d'un rang de fausses perles.» (Morand 1992: 126) [これはエゴイズムと絲瓜の水とのお蔭で殘りの色香まだ全く消えやらぬ一人の小造 りな女である。かの女は 顔の皺を片づけて皆あつめて耳の後で 結んで置いた。か の女の胸には人造真珠の首環の一列が葑いたやうに散らばってみた。] (Horiguchi 1986: 270) 
Dans la traduction, Horiguchi a ajouté la description «殘りの色香まだ全く消え やらぬ» [dont la couleur et le parfum restés ne disparurent pas encore entièrement] en plus de «le lait de concombres et l'égoïsme» [エゴイズムと絲瓜の水]. D’ailleurs, en traduisant l'adjectif «ensemencée» qui est une métaphore dans le texte, il a ajouté un verbe comme véhicule de la métaphore pour enrichir sa figuration. Dans le texte original, «ensemencée» sert à décrire le fait que les fausses perles semblent jaillir de la poitrine de la mère d'Isabelle, alors que dans la traduction, Horiguchi a gardé le sens d'ensemencer en utilisant le verbe japonais «蒔く», puis il a ajouté le verbe 《散らす» [disperser] pour comparer la disposition de fausses perles sur la poitrine à celle d'une rangée de plants. Donc, avec ces deux verbes: «蒔く» [ensemencer] et 《散らす» [disperser], le sens du texte traduit est quelque peu manipulé: de «un rang de fausses perles ensemencées» à "un rang de fausses perles dispersées comme des plants ensemencés rang par rang». L'image de fausses perles devient plus riche et figurative. Ces descriptions ajoutées au dialogue de la mère d'Isabelle et à sa tenue vestimentaire soulignent davantage son allure mondaine et matérialiste. Pareillement, Horiguchi a mis l'accent sur l'image indépendante, voire excentrique d'Isabelle:

Elle [Isabelle] passe sa vie à se satisfaire follement comme dans les rêves. (Morand 1992: 126)

あれは只もう自分を滿足させることに許り齓齪してまるで夢でも見てみる人のやうにし てその日その日を暮して行くのです。 (Horiguchi 1986: 271).

Le traducteur a souligné l'esprit révolté de l'héroïne par l'ajout d'un détail, le fait qu'elle s'empresse [許り齓齪して] de se satisfaire jour par jour [その日その日を]. Comme l'image mondaine de la mère et l'image émancipée de la fille forment un contraste plus violent, le lecteur est amené à croire que la mère représente peut-être la vieille génération corsetée tandis que la fille représente une nouvelle génération, caractérisée par un désir de libération individuelle et qui cherche toujours à jouir de la vie et à en comprendre le sens.

Dans la seconde nouvelle intitulée La nuit des six-jours, Horiguchi a également manipulé l'image par l'ajout de descriptions. Outre la rencontre entre le narrateur et la jeune fille Léa, typique du schéma narratif morandien, la scène du concours de six-jours est l'autre événement attrayant de l'histoire. Afin de rendre sensible au lecteur l'atmosphère surchauffée du concours, Horiguchi a interprété la métaphore du texte original «À l'emballage, ils s'abattirent sur la prime comme des carpes sur un quignon.» (Morand 1992: 140) comme suit: [決勝線に近づくと競争者は麩のまは りに集まる鯉のやうにかたまって互に先を爭って雪崩れこんだ。] (Horiguchi 1986:285)

Alors que dans le texte original, Morand a utilisé la métaphore «des carpes sur un quignon» pour décrire l'environnement compétitif au sein duquel évoluent les joueurs de six-jours, Horiguchi a traduit «des carpes»par 《集まる鯉のやうに» [comme des carpes qui se réunirent], et «s'abattirent sur la prime» par «互に先を爭 つて雪崩れこんだ» [s'abattirent et se précipitèrent sur la prime]. Ainsi, par ses ajouts, l'action de s'abattre est dynamisée et accentuée.

Si l'ajout de descriptions pour clarifier le texte traduit est une démarche courante dans la traduction, la personnification ajoutée dans la traduction des nouvelles de Morand est davantage révélatrice de la volonté d'Horiguchi de mettre en valeur la dynamique et la vivacité des images et des métaphores du texte original. Par exemple, les descriptions des sonneries et de Paris chez La nuit catalane (1922): «Des sonneries 
grelottaient. Les voyageurs coulaient sur l'asphalte.» (Morand 1992: 77) traduit [電 鈴がわなないた。アスファルトの上を旅客たちが流れた。] (Horiguchi 1986: 5) et l'exemple suivant:

Paris fut débordé par les pluies d'un novembre amolli où, dans l'asphalte, les maisons étendaient leurs reflets jusqu'au toit. Les embruns autour des réverbères délayaient une poudre rose. (Morand 1992: 97)

巴里わ溶けるやうなその年の十一月がふらした雨で溢れていた、街のアスファルトの上 には両側の家々の影が屋根まではっきりと映ってるた。街頭の灯を周る霧の中には桃 色の微粉末が踊ってなた。(Horiguchi 1986: 61)

Dans le premier exemple, le verbe «grelotter» peut signifier «être agité de tremblement sous l'effet d'une très vive sensation de froid» ou, plus littérairement, «faire entendre un bruit de grelots». Horiguchi a choisi le premier sens en traduisant le verbe français par «戦慄く», c'est-à-dire trembler; les sonneries sont donc personnifiées. Dans ce premier exemple, le choix du traducteur pourrait s'expliquer par un choix portant sur le sens du mot. En revanche, le deuxième exemple est, à l'évidence, la preuve d'une volonté, de la part d'Horiguchi, d'ajouter une personnification. Ce deuxième exemple est une description composée d'une série de métaphores sur Paris sous les averses. Dans la rue, les lumières des réverbères se reflètent dans les gouttes de pluie. Le verbe «délayer» signifie «incorporer» ou "mélanger» et Horiguchi l'a traduit «踊ってみた»(danser en forme passé) pour décrire la scène. Dans la version de ce dernier, avec le verbe «danser», la scène devient: les embruns roses de la pluie dansent autour des réverbères [街頭の灯を周る霧の中には桃色の微粉末が踊ってみ た], c'est-à-dire que les gouttes sont personnifiées. Ces deux exemples comptent parmi les nombreux ajouts de personnifications par Horiguchi, là où le texte original n'en comporte pas, dans le but de rendre le style du texte de Morand plus lyrique ${ }^{19}$.

En réalité, dans sa première traduction des nouvelles de Morand, Horiguchi a déjà tenté de souligner le style nouveau et expérimental de ce dernier qui, pour lui, réside dans l'emploi de métaphores et d'images originales. Au fil de sa traduction de Morand, Horiguchi s'est donc attaché à mettre en valeur la dynamique et la vivacité des images et des métaphores du nouvelliste français. Il a presque adopté une démarche inverse à celle de Marcel Proust qui dans sa préface au premier recueil de nouvelles de Morand Tendres Stocks (1921), a critiqué l'excès d'images et de métaphores ${ }^{20}$.

\subsection{Le naturalisme, le modernisme et le style}

Comme les chercheurs japonais tels que Wananabe Kazutami, Tsuchiya Satoshi et Ōmura Azusa l'ont déjà remarqué, Horiguchi n'était guère un traducteur fidèle des nouvelles de Morand. Wananabe ne l'a mentionné que très brièvement, alors que Tsuchiya a mis en doute la fiabilité de la traduction d'Horiguchi en constatant que ce dernier ne maîtrise pas parfaitement la langue française: malgré la pratique du français oral avec sa belle-mère belge, il n'a cependant séjourné en tout et pour tout que deux mois en France. Par ailleurs, comme la plupart des Japonais de l'époque, le français qu'il a appris était essentiellement livresque (Tsuchiya 2006, 87-102). Par l'analyse comparative entre les textes-sources et les textes-traduits, Ōmura a conclu qu'Horiguchi avait transformé quelques passages descriptifs afin de rendre les images de Morand plus «nouvelles» (Ōmura 2011 : 92-102). À ce titre, il conviendra d'appro- 
fondir la question en nous interrogeant sur la signification des manipulations du texte-source par Horiguchi. Quel est le rapport entre le style métaphorique de Morand et le modernisme de la littérature japonaise dans le contexte littéraire des années 1920 ? Pourquoi est-ce plutôt le style de Morand qui est considéré comme un élément essentiel à la pratique moderniste de l'École des sensations nouvelles. Pour répondre à ces questions, il faut remonter un peu le cours de l'histoire littéraire japonaise jusqu'aux années 1900. Entre les premières années du siècle et le début des années 1920, le naturalisme japonais et sa variante le Shishōsetsu (私小説) ${ }^{21}$ dominaient le monde littéraire japonais. Le «Shishōsetsu» est désigné par les caractères «je» (shi) et "roman" (shōsetsu): sa traduction française est donc "roman-je». Il est relaté à la première ou à la troisième personne, de manière à mettre en lumière l'engagement absolu de l'auteur dans le récit. Pour les naturalistes japonais, l'écrivain doit être proche de la «nature» et la valeur littéraire n'apparaît qu'à travers l'écriture de «la vérité». Le mot nature (自然) en japonais peut signifier ce qui est naturel, véridique, tout ce qui nous arrive naturellement appartient à la nature, tels que les sentiments, les désirs. C'est pourquoi le premier écrivain japonais à être appelé naturaliste, Kosugi Tengai (1865-1952), a écrit: «la nature est la nature, celle-ci n’est ni bonne ni mauvaise, ni belle ni laide ${ }^{22}$ ». Selon cette définition de «la nature», les écrivains naturalistes sont tenus de reproduire exactement les événements auxquels ils ont été confrontés dans la vie quotidienne. Les détails des faits doivent être restitués. Citons la parole d'un autre naturaliste représentatif, Tayama Katai (1872-1930) pour expliciter cette idée: «décrire tous les phénomènes comme ils sont entendus, vus ou touchés ${ }^{23}$.» Cette démarche fait écho à celle de Zola: «la reproduction exacte de la vie» (Cogny 1976: 4). Cependant, dans la variante du naturalisme japonais, le Shishōsetsu, [la reproduction de la vie]a atteint un niveau extrême, au point qu'on exige une représentation directe des impressions et des descriptions subjectives, comme Shimazaki Tōson (1872-1943) l'a souligné: «[je] n’écris pas ce qui s'est passé à l'extérieur, tout se limite à la scène de la maison. J'écris la cuisine, puis la porte, ensuite le jardin de la maison. Si cette maison peut faire entendre le bruit de l'eau, j'écrirai la rivière ${ }^{24}$." Autrement dit, il n'écrit que les phénomènes comme ils sont entendus, vus ou touchés à l'intérieur de la maison (la famille). Ainsi, «la vérité» n’est présente qu'au sein des expériences vécues par l'auteur, ou même se limite aux expériences personnelles de l'auteur à l'intérieur de la sphère familiale. À partir de cette notion de la vérité, les naturalistes japonais excluent certaines techniques littéraires destinées à imiter au mieux «la nature», parce qu'à leurs yeux, elles risqueraient d'incorporer des valeurs et des jugements de valeur à leur description. Autrement dit, les naturalistes japonais rejettent l'imagination, la fiction et le style dans leur pratique de la littérature.

En rupture avec cette notion puissante du renoncement au style, en vogue au début des années 1920, Horiguchi a souligné le style métaphorique novateur de Morand en avançant l'hypothèse que ce dernier aurait créé de nouvelles connexions entre les objets en partant d'une «logique de la sensation» (Horiguchi 1986: 220). Celle-ci, aux yeux d'Horiguchi, est une logique qui n'est pas fondée sur la construction d'un récit, autrement dit sur la restitution d'un enchaînement cohérent de faits, mais sur l'évocation de correspondances que l'esprit opère de manière instantanée entre le monde extérieur et son ressenti, créant ainsi «un monde de sensations». Certes, Morand a employé des images purement visuelles qui résistent souvent à l'analyse et à la logique rationnelle. Par exemple, dans La nuit de Portofino Kulm 
$(1923)^{25}$, le narrateur se promène dans un village de la Riviera italienne. Il s'aperçoit que le ciel étroit «s'allonge» (Morand 1992: 206) entre les maisons, encadré "par des fumées arborescentes qui poussaient hors des cheminées» (Morand 1992: 206). Le soleil frappe une vitre et, «décontenancé par ce premier choc» (Morand 1992: 206), vient par ricochet «s'abattre» (Morand 1992: 206) à ses pieds. Un peu plus loin, le narrateur remarque une terrasse inondée de lumière rose, contrastant avec les taches sombres des chambres, "fraîches mariées derrière leurs moustiquaires» (Morand 1992: 207). Près de la porte se tient un palmier au tronc natté, "poilu comme un chimpanzé» (Morand 1992: 208), auquel pend, un «fruit indigène» (Morand 1992: 208), un thermomètre. Les descriptions de Morand ici sont fondées sur une logique purement visuelle, l'écrivain propose au lecteur «une série de trompe-l'œil, d'illusions d'optique et de couleurs qui créent un espace nouveau et déconcertant» (Thibault 1992 : 21). C'est pourquoi Horiguchi a avancé l'hypothèse que Morand avait fondé la cohérence de son style littéraire sur une «logique de la sensation».

L'écriture de la sensation n'est pas une entreprise littéraire novatrice. Si nous nous intéressons à l'histoire de la littérature japonaise, nous pouvons facilement trouver la description des sensations ou une forme d'écriture sensible dans la littérature traditionnelle. Cependant, considérer la sensation comme un mode d'expression littéraire privilégié, exigeant de la part du lecteur une attention soutenue aux figures de style (ellipses, parataxes, zeugmes, etc.), aux idiolectes (néologismes, mots étrangers) et à l'aspect inhabituel et pléthorique des métaphores employées (Douzou 2003 : 38) est un parti pris rare. Comme nous venons de l'analyser, Horiguchi a exacerbé par sa traduction du style imagé et suggestif de Morand en ajoutant des descriptions et des personnifications dotées d'un effet hyperbolique. Par conséquent, sa traduction d'Ouvert la nuit a été un choc immense au sein de la communauté littéraire, alors dominée par le naturalisme et sa variante le Shishōsetsu, surtout pour les jeunes lecteurs qui remettaient en cause le style sec et littéral du naturalisme.

Si le style métaphorique de Morand a choqué le monde littéraire japonais, «la logique de la sensation» employée par Horiguchi pour comprendre l'horizon littéraire morandien est le véritable enjeu qui a élevé l'écrivain français au rang de modèle aux yeux des membres de l'École des sensations nouvelles, plus globalement aux yeux des tenants du discours moderniste japonais. En effet, Morand et l'École des sensations nouvelles partagent en commun cette conception esthétique de «la sensation » et placent l'intuition sensible avant la pensée rationnelle. Dans le discours de l'École des sensations nouvelles, la «sensation nouvelle» est une forme symbolique d'expression. Elle est associée aux procédés littéraires utilisés pour construire un sens hautement symbolique et mystérieux exigeant de la part du lecteur une attention et une complicité particulières. L'écrivain adhérant à ces principes esthétiques s'appuie sur des métaphores et des symboles. Comme Chiba Kameo l'a mentionné dans «La naissance de l'École des sensations nouvelles»:

Nous pourrions actuellement appeler notre époque «l'époque de sensations nouvelles». Les auteurs se tiennent au sommet d'un champ visuel spécial et s'en délectent, regardant avec un regard qui dévoile tous les aspects cachés de la vie [...] ils nous permettent de scruter la vie intérieure dans la totalité de son existence et de son sens par de petits trous [...]. L'arrivée d'un art si subtil est la promesse de la nature. Pourquoi, dans leur expression de la vie, doivent-ils ouvrir ces petits trous? Parce que ce n'est rien de plus que la sensation immédiate de stimulations extrêmes qu'ils utilisent pour symboliser 
cette grande vie intérieure à travers ces formes et figures, petites et externes. (Chiba 1956-1957, 193-196; traduction de l'auteur de l'article $)^{26}$

Dans ce passage et d'autres passages de Chiba, de "petits trous» (Chiba 1956$1957,194)$ nous aident à «scruter la vie intérieure dans la totalité de son existence et de son sens» (Chiba 1956-1957, 194). Ces «petits trous» évoquent en fait le style d'écriture des écrivains appartenant à l'École des sensations nouvelles. Il s'agit d'une nouvelle langue aiguë et audacieuse, aux descriptions et métaphores très vives. Grâce à ce style, le lecteur peut se représenter les sensations instantanées procurées par un fragment de réalité, c'est-à-dire un instant particulier. D'une certaine manière, ces images véhiculées dans le texte permettent au lecteur d'éprouver (par l'ouïe, la vue, le goût, etc.) les réalités décrites; elles facilitent donc une approche subjective des œuvres textuelles et, par extension, de la conscience moderne et des sensations. En même temps, «la logique de la sensation» d'Horiguchi, qui, dans un certain sens, ne cherche pas à raconter une histoire, mais à évoquer les émotions et les associations de la pensée à un instant précis, est conforme à la théorie de l'École des sensations nouvelles. Donc, dès lors qu'Horiguchi a proposé de passer par le prisme de «la logique de la sensation" pour percevoir le monde littéraire de Morand, ce dernier a toujours été lié au mot "sensation» et à l’École des sensations nouvelles dans les ouvrages critiques, telle que 新感覺派及びモオランの『夜ひらく』に就て [À propos de l'École des sensations nouvelles et Ouvert la nuit de Morand, 1925] (Nakamura Kan'ichi 1925/1980, 227-238).

Outre les critiques japonais, Liu Na’ou qui était très important pour la réception de Morand en Chine, a aussi employé «la sensation nouvelle» comme un important critère dans sa lecture de l'œuvre de Morand. Après avoir achevé la lecture d'un recueil de poèmes de Morand au milieu des années 1920, il fit une remarque en employant le mot «sensation»: "Bien que la tonalité moderniste soit très forte, elle est superficielle: il y a seulement quelques mots qui ont la couleur de la sensation nouvelle 27 ." De ce point de vue, le fait d'être associé à la «sensation» a placé Morand du côté de l'École des sensations nouvelles ainsi que des idées modernistes dans le contexte littéraire japonais. Dès lors, ce n'est pas seulement parce que Morand a adopté un style métaphorique suffisamment puissant pour ébranler les jeunes lecteurs qu'il a pu s'imposer dans le discours du modernisme japonais, c'est aussi sa «logique de la sensation» présentée telle quelle par Horiguchi qui a définitivement associé l'auteur français au mouvement moderniste des années 1920.

\section{Traduction chinoise des œuvres de Morand}

À travers l'introduction de l'École des sensations nouvelles japonaise, la vénération pour Morand a également attiré l'attention de Liu Na'ou au milieu des années 1920, lorsqu'il faisait ses études au Japon. Après son retour en Chine, Liu a participé à la dissémination de la culture moderniste à Shanghai. En 1928, Liu a dirigé la revue moderniste 無軌列車 [Train sans chemin] dont le quatrième numéro, consacré à Morand, présente deux nouvelles de l'écrivain français: «Vague de paresse» (1925) et «Les amis nouveaux» (1925), traduites par Dai Wangshu, ainsi qu'une version traduite de l'article «Paul Morand» de Benjamin Crémieux (1888-1944) (1924) ${ }^{28}$. Grâce à ce numéro spécial, Morand est officiellement entré dans l'horizon de la littérature moderniste chinoise $\mathrm{e}^{29}$. 
Tandis que le lien entre Morand et le modernisme japonais avait été établi par le biais de l'École des sensations nouvelles japonaise, considérée comme le premier courant moderniste dans le domaine de la fiction au Japon, dès les premiers moments de sa réception en Chine, il avait déjà été classifié comme écrivain moderniste. Morand a été considéré moderniste parce que son image au Japon était étroitement liée à l'École des sensations nouvelles japonaise, son intermédiaire chinois Liu Na'ou, son traducteur principal Dai Wangshu, et la revue littéraire [Train sans chemin], représentaient les figures de proue du modernisme chinois. Dans la postface du quatrième numéro de [Train sans chemin], l'éditeur Liu Na’ou a même affirmé que Morand était le pionnier de l'art avant-gardiste.

Même si le rapport entre Morand et le modernisme chinois est évident, nous creusons encore le lien entre la stratégie de traduction et la modernité chinoise dans les années 1930. Dans cette partie, nous suivons, en partie, la chronologie des traductions japonaises des œuvres de Morand en divisant la traduction chinoise en trois parties: la vie du traducteur principal chinois des œuvres de Morand, sa stratégie de traduction - la traduction littérale - et le rapport entre cette stratégie et la modernité chinoise.

\subsection{Dai Wangshu: moderniste et traducteur}

Parmi les traducteurs chinois des œuvres de Morand, Dai Wangshu a traduit la plupart des nouvelles de Morand et les a recueillies dans une collection nommée 天女 玉麗 [Céleste Julie] (1929), dont les nouvelles proviennent principalement d'Ouvert la nuit et de l'Europe galante (1925). En comparant les traductions chinoise et japonaise des œuvres de Morand, les premières sont moins riches que les secondes, tant en quantité qu'en diversité de genres ainsi que le montre le tableau ci-dessous:

\section{TABleAu 2}

La traduction chinoise des œuvres de Paul Morand entreprise par Dai Wangshu pendant les années 1920 et 1930

\begin{tabular}{|c|c|c|}
\hline Nom original de l'œuvre & Nom chinois de l'oeuvre & Ressource chinoise \\
\hline $\begin{array}{l}\text { «La nuit des six-jours». } \\
\text { In: Ouvert la nuit (1922) }\end{array}$ & 〈六日之夜〉 & $\begin{array}{l}\text { 《法蘭西短篇傑作集》(第一集) } \\
\text { (Chef'd'euvre des nouvelles françaises, } \\
\text { vol.1) }(1928)^{30}\end{array}$ \\
\hline $\begin{array}{l}\text { «Les amis nouveaux». } \\
\text { In: L'Europe galante (1925) }\end{array}$ & 〈新朋友們〉 & $\begin{array}{l}\text { 《天女玉麗》(1929) } \\
\text { (Recueil de nouvelles de Morand, le } \\
\text { traducteur a repris le titre de la nouvelle } \\
\text { «Céleste Julie», incluse dans le recueil) }\end{array}$ \\
\hline $\begin{array}{l}\text { "Céleste Julie». } \\
\text { In: L'Europe galante (1925) }\end{array}$ & 〈天女玉麗〉 & 《灭女玉麗》[Céleste Julie, 1929] \\
\hline $\begin{array}{l}\text { «Le musée Rogatkine». } \\
\text { In: L'Europe galante (1925) }\end{array}$ & 〈洛迦特金博物館〉 & 《天女玉麗》 [Céleste Julie, 1929] \\
\hline $\begin{array}{l}\text { «La nuit des six-jours». } \\
\text { In: Ouvert la nuit (1922) }\end{array}$ & $\langle$ 〈六日競走之夜〉 & 《天女玉麗》[Céleste Julie, 1929] \\
\hline $\begin{array}{l}\text { «Vague de paresse». } \\
\text { In: L'Europe galante (1925) }\end{array}$ & 〈懶惰底波浪〉 & 《天女玉麗》[Céleste Julie, 1929] \\
\hline $\begin{array}{l}\text { «Madame Fredda». } \\
\text { In: L'Europe galante (1925) }\end{array}$ & 〈茀萊達夫人〉 & 《天女玉麗》[Céleste Julie, 1929] \\
\hline
\end{tabular}




\begin{tabular}{|c|c|c|}
\hline $\begin{array}{l}\text { "La nuit hongroise». } \\
\text { In: Ouvert la nuit (1922) }\end{array}$ & 〈匈牙利之夜〉 & 《天女玉麗》[Céleste Julie, 1929] \\
\hline $\begin{array}{l}\text { "La nuit romaine». } \\
\text { In: Ouvert la nuit (1922) }\end{array}$ & 〈羅馬之夜〉 & $\begin{array}{l}\text { 《法蘭西現代短篇集》 } \\
\text { Les nouvelles modernes de la France (1934) }\end{array}$ \\
\hline
\end{tabular}

Dai Wangshu a découvert sa passion pour l'écriture et l'édition durant ses deux dernières années de lycée. Son coup de foudre pour la littérature française a lieu entre 1922 et 1924, période au cours de laquelle il manifeste un intérêt croissant pour les ouvrages étrangers, en particulier la littérature française. Cet intérêt le pousse à s'inscrire à l'Université Aurore de Shanghai, une institution jésuite proposant un cours spécial et intensif de français focalisé sur la lecture de textes littéraires. De ce fait, la capacité de lecture de Dai était bonne mais sa maîtrise de la langue parlée était limitée au début. En août 1928, Dai publie six poèmes importants dans la revue 小說月報 [Roman mensuel]. L'un d'entre eux, 雨巷 [L'allée de la pluie] a été une source d'inspiration pour les jeunes lecteurs de l'époque, qui ont été séduits par son style et son caractère symboliste. Ce poème a aussi permis aux lecteurs chinois d'identifier Dai au modernisme chinois. Dai a donc établi sa réputation de poète moderniste, jouissant d'une certaine renommée à la fin des années 1920.

En ce qui concerne la traduction, Dai préférait la traduction littérale pendant les années 1920. L'anecdote suivante montre son purisme en matière de traduction: Li Sichun (date inconnue), un professeur de littérature française de l'Université du SudEst avait traduit Le printemps de Charles d'Orléans (1394-1465) ${ }^{31}$ et l'avait publié dans une revue trimensuelle 瓔絡 [Collier de jade] ${ }^{32}$, mais Dai a trouvé sa traduction inexacte. Il a donc retraduit ce poème:

Le Printemps de Charles d'Orléans, [Collier de jade], numéro 2 (1926), p. 13-14.

Le temps a laissé son manteau

De vent, de froidure et de pluie

Et s'est vêtu de broderie

De soleil rayant, clair et beau (Charles D’Orléans 1923-1924: 307)

La traduction de Li (1926):

風微雨歇嚴凍解。春已脫袍露真面。別換新妝繡。日光澄澈明而灎。

[Le vent faiblit, la pluie s'arrête, la froidure se libère. Le printemps se débarrasse de son manteau en dévoilant son vrai visage. Il se pare d'un nouveau vêtu de broderie. De soleil clair, lumineux et rayonnant]. (Notre rétrotraduction)

La traduction de Dai (1926):

時間已棄了他的風、寒、雨大衣, 而穿起了光耀而明麗的太陽的繡衣。(Lee 1989: 5)

[Le temps a laissé son manteau de vent, de froidure et de pluie, et s'est vêtu de broderie de soleil rayant, clair et beau]. (Notre rétrotraduction)

À l'évidence, la traduction de Li (1926) revêt une allure classique, mais sacrifie la fidélité au texte-source. Ce dernier a emprunté certaines règles des poésies classiques chinoises telles que l'usage de cinq ou sept caractères par vers ainsi que l'emploi d'un vocabulaire et d'une formulation caractéristiques de la poésie classique [風微雨歇嚴凍解], [新妝繡]. Cependant, il a également contourné ces règles traditionnelles comme le montrent la combinaison inédite des tons (平人) et le parallélisme (對仗); le troisième vers ne s'identifie pas non plus aux trois autres vers, car il ne comprend que cinq caractères tandis que ces derniers ont sept caractères. Donc 
sa traduction est chancelante: demi-chinoise et demi-européenne, semi-classique et semi-moderne. Comparée à la traduction de Li (1926), la traduction de Dai (1926) est entièrement littérale. Elle ne parvient donc pas à restituer le caractère poétique du texte-source.

À travers l'étude de différentes méthodes employées par Li et Dai pour traduire un texte, nous faisons l'expérience de conceptions très diverses de la traduction à l'œuvre durant cette époque. En réalité, trente ans avant la traduction de Li et Dai, époque durant laquelle la littérature française a été introduite en Chine pour la première fois, les traducteurs chinois, représentés par Linshu (1852-1924), adoptaient souvent «la traduction impressionniste». Linshu ne connaissait aucune langue étrangère, mais il a traduit en chinois plus de 180 genres provenant de la littérature étrangère, dont les romans anglais, français, américain, russe, grec, japonais faisaient partie. Le premier roman français traduit en chinois, La dame aux camélias ${ }^{33}$ (1848, traduit en chinois en 1898) d'Alexandre Dumas fils (1824-1895), a été son œuvre. Lors de la traduction, Linshu a collaboré avec un expert français qui lui relatait l'histoire que Linshu réécrivait en chinois lyrique. Par cette approche «impressionniste» de la traduction et en collaborant avec d'autres experts en langues étrangères, Linshu a fait connaître aux lecteurs chinois de nombreux grands écrivains étrangers comme Shakespeare (1564-1616), Charles Dickens (1812-1870), Victor Hugo (1802-1885), Henrik Ibsen (1828-1906), etc. Son style est encore marqué par l'usage du chinois classique, mais est délibérément moins métrique pour rendre les textes traduits plus lisibles (Guo Yanli 1997: 264-271).

Les générations de traducteurs allant de Linshu à Dai Wangshu, en passant par Wu Guangjian (1867-1943) et Zengpu (1871-1935) également considérés comme des traducteurs importants de la littérature française, réalisent une avancée dans leur champ disciplinaire, à partir de la traduction impressionniste (Linshu), en passant par la traduction libre, cette dernière rejetant les descriptions détaillées (Wu), jusqu'à la nouvelle importance accordée à la fidélité au texte-source (Zen et Dai). L'emploi du chinois vernaculaire au sein de la traduction a également supplanté celui du chinois classique. Cette évolution a eu lieu en seulement trente ans, c'est pourquoi les partis pris méthodologiques ou les discours dominants à l'œuvre dans la traduction d'aujourd'hui, telle que la fidélité au texte-source, ne sont pas encore bien établis à l'époque de Li et Dai qui adoptaient des méthodes très différentes de la traduction pour le même poème. L’un utilisait encore la méthode traditionnelle et un style classique (Li), l'autre est à la fois le disciple de la culture moderniste et de la traduction littérale qui avaient cours pendant les années 1920 (Dai).

\subsection{Stratégie de traduction: la traduction littérale}

Dai Wangshu a une fois de plus montré sa préférence pour la littéralité dans sa traduction du recueil de nouvelles de Morand. La traduction est principalement proche du texte-source, dans le sens où il n'a pas seulement traduit le contenu du textesource, mais en a également respecté la syntaxe, la grammaire, la ponctuation ainsi que les expressions vernaculaires françaises. Par exemple, pour un passage de La nuit des six-jours, la traduction chinoise de Dai imite l'ordre des signes de ponctuation ainsi que l'ordre d'enchaînement des adjectifs du texte-source. 
Quand on l'invitait, elle refusait: moi comme les autres, bien que je fusse venu pour elle, et elle le savait. Ce n'était pas son dos lacté, sa robe de jais, tremblante pluie noire, un excès de bijoux d'onyx, dont des yeux étirés et, noués aux guignes de l'oreille; c'était plutôt son nez aplati, le bondissement de sa poitrine, son beau teint juif de vigne sulfatée, cet isolement un peu louche. (Morand 1992: 137)

當別人邀請她的時候, 她辭絕了; 我也正和別人一樣地被她辭拒, 雖然我是為她而來, 而這個她又是知道的。我來的目的並不是為了她的乳白的背, 她的黑玉的衫子一一戰 慄著的黑雨, 無數的縞瑪瑙的珍飾(從這些珍飾間是可以辨出細長的, 和耳邊卷髮相 接的眼睛來的); 卻可說是為了她的扁平的鼻子, 她的突起的胸膛, 她的像灑過硫酸鹽 的葡萄葉的, 美麗的猶太風的顏色, 她那個有些蹊蹺的孤獨癖。(Muhang

[Morand]1929: 53-54, traduction de Dai)

La traduction chinoise a entièrement respecté la syntaxe française, surtout dans la phrase «son beau teint juif de vigne sulfatée». Cette syntaxe est très rare dans la langue chinoise, car il y a trop d'adjectifs. Et pour permettre l'enchaînement de ces trois adjectifs «beau teint», «teint juif», «vigne sulfatée» dans la phrase, Dai a utilisé cinq 《的» (de en français): [她的像灑過硫酸鹽的葡萄葉的, 美麗的猶太風的顔色], tandis que la structure de la phrase chinoise comprend en général seulement un ou deux adjectifs tels que «son dos lacté» traduit par [乳白的背] ou de «son nez aplati» [扁平的鼻子]. Dai a cependant gardé cette partie de la phrase comportant beaucoup d'adjectifs dans une phrase courte, créant ainsi ce que l'on considère comme un style européanisé.

Si l'exemple ci-dessus montre la stratégie de la traduction littérale de Dai, le sens du passage traduit reste par ailleurs compréhensible, l'exemple suivant montre qu'il n'a parfois pas respecté le sens du texte-source pour garder la syntaxe française. Dans la scène où le narrateur rencontre la protagoniste de La nuit hongroise (1922), «À préférer celle-ci, je sentais cette supériorité aisée des rêves, et comme en rêve, je fus puérilement heureux de pouvoir dire: [...]» (Morand 1992: 150) traduit ainsi par Dai: 《在選中這一個的時候, 我感到這種夢的容易的優越, 而且, 像在夢中一般地, 我是像孩子一樣地快樂可以這樣說 […]» (Muhang 1929: 95, traduction de Dai)

Dans la dernière partie de ce passage: «je fus puérilement heureux de pouvoir dire» [我是像孩子一樣地快樂可以這樣說], Dai utilise [是] et [像孩子一樣地] pour traduire les mots «fus» et "puérilement», commettant sciemment une faute de grammaire: 是 [fus] étant superflu. De plus, le sens de la phrase est «je fus puérilement heureux» parce que «je pus dire [quelque chose] », la conjonction «de» énonce une causalité, mais la traduction chinoise ignore cette causalité et la traduit directement, en conséquence, la phrase traduite devient une phrase grammaticalement fautive et sémantiquement confuse. Une traduction plus appropriée pourrait être: 我是像孩子一樣地快樂 (, 因為我) 可以這樣說). Ainsi, ce genre de traduction littérale arrive-t-elle au niveau de [la traduction raide] (硬譯).

À cause de la traduction littérale, certaines phrases traduites comportent trop d'adjectifs, de fautes de grammaire chinoise, de confusions sémantiques, ou sont trop longues, comme celles-ci: 《另一個女子是穿著瘦的筋肉, 科的靜脈, 手鐲的緊 合, 骨的影和一個神聖的獸的臉兒的》 (Muhang 1929: 95, traduction de Dai) et 《在 那映出櫻桃色的花鍛片和模仿匈勃朗寶殿的式樣的古式廊的威尼市的影子的中間》 (Muhang 1929: 97, traduction de Dai). Ce type de phrase est fréquent dans la traduction de Dai, ce qui rend la compréhension parfois difficile. Néanmoins, dans sa propre écriture, Dai n'utilise absolument pas ce type de langue chinoise. Par exemple, dans 
ses nouvelles de la fin des années 1920, contemporaines de ses traductions des nouvelles de Morand, ainsi que dans ses récits de voyage en Espagne publiés au début des années 1930, il se livre comme Morand à une description des villes qu'il traverse ainsi qu'à un portrait des femmes qu'il rencontre. Il emploie un registre de langue à la fois poétique et empreint d'une grande clarté ${ }^{4}$. Il existe donc un grand décalage entre le langage de sa propre écriture qui est limpide et pleine de beauté, et le langage de ses traductions qui est lourd et parfois difficile à comprendre. Pourquoi cette grande différence? Cela touche sans doute au processus de construction de la langue moderne chinoise à travers langage de la traduction.

\subsection{La traduction littérale et la modernité de la langue chinoise}

Le chinois moderne est officiellement marqué par le Mouvement du chinois vernaculaire (白話文運動, 1919) ${ }^{35}$ et il s'est formé principalement à partir du chinois classique, des langues européennes et du chinois parlé. La langue vernaculaire existait en Chine depuis longtemps, employée à titre d'exemple dans le Huaben (話本) ${ }^{36}$ qui est un genre de conte écrit en langue vulgaire, datant des dynasties Tang (618-907) et Song (960-1279), ou dans la fiction (小說) écrite en chinois vernaculaire classique, datant des dynasties Ming (1368-1644) et Qing (1636-1912) dont les œuvres emblématiques sont 水澈傳 [Au bord de l'eau $]^{37}$, ou encore 西遊記 [La pérégrination vers l'Ouest $]^{38}$. Mais selon la hiérarchie des genres littéraires classiques, la fiction n'était pas considérée comme de la littérature. La fiction a donc eu une influence limitée et son usage ne s'est pas érigé en modèle. Sous la période du Mouvement du chinois vernaculaire dans les années 1910, l'usage du chinois vernaculaire était cependant devenu une étape importante dans la modernisation de la Chine.

Les premiers partisans de ce mouvement croyaient que la syntaxe des langues européennes était logique, appropriée au discours argumentatif, et donc caractéristique de l'esprit moderne. Par conséquent, ils voulaient introduire la syntaxe, la grammaire et le vocabulaire des langues européennes dans le chinois moderne à travers la traduction. Leur but était d'établir un standard esthétique et de codifier le chinois moderne à partir de la logique sous-tendant les langues européennes. Certains intellectuels pensaient que la traduction littérale était la meilleure démarche pour parvenir à cette fin. Par exemple, le géant de la littérature moderne chinoise et grand traducteur Lu Xun (1881-1936) était un fervent adepte de la traduction littérale. D’une part, il adhérait au principe de fidélité au texte-source en pratiquant un littéralisme extrême dans ses traductions. Ses traductions, en particulier, des œuvres littéraires russes et des critiques littéraires marxistes à la fin des années 1920 sont un bon exemple de son littéralisme extrême. D’autre part, il voulait transformer le chinois en langue plus subtile, plus logique. Les traductions de Lu Xun ont été ainsi souvent critiquées en raison de son non-respect du sens du texte traduit. L'historien Fu Sinian (1896-1950) partageait le même avis que Lu Xun: il pensait que l'une des deux approches pour fonder une langue chinoise moderne était d'y transposer la syntaxe et la grammaire européennes. Il a affirmé:

On utilise directement la forme, la grammaire, la morphologie, la composition du texte, le vocabulaire [...] toutes les méthodes de la rhétorique européenne, en créant une langue nationale qui dépassera la langue nationale existante. Ce serait une langue 
nationale européanisée (歐化國語), et la littérature employant cette langue serait une littérature nationale européanisée. (Fu Sinian 1919/1935: 223, notre traduction) ${ }^{39}$

Fu Sinian mentionne ici l'un des buts du Mouvement du chinois vernaculaire: construire l'identité nationale à travers une langue moderne, accessible au grand public (Hu Shi 1918/1996: 77-102). C'est la conception de la langue nationale (國語). Par conséquent, l'identité nationale moderne ne peut advenir que par l'usage d'une langue moderne. De la même manière que Lu Xun et Fu Sinian, le poète Zhu Ziqing (1898-1948) pensait qu'il ne faut pas se limiter au chinois vernaculaire classique, ni au chinois parlé, il faut absorber la langue vernaculaire des textes traduits ${ }^{40}$.

Les lumières de ces intellectuels nous permettent de mieux comprendre pourquoi Dai Wangshu préférait la traduction littérale dans les années 1920 et 1930 même s’il ne fait guère référence à la théorie de la traduction elle-même. Mais comme la plus grande partie de l'œuvre de Dai est constituée de poèmes, l'influence de sa traduction littérale de Morand sur sa création n'est pas évidente. Elle est plus perceptible dans les nouvelles de Liu Na'ou. En comparant les nouvelles de Liu et la traduction chinoise des nouvelles de Morand, nous relevons dans l'écriture de Liu une imitation de certaines images, mots et syntaxe présents dans les nouvelles de Morand. Ci-dessous, nous avons pris La nuit de six-jours comme exemple:

\section{TABLEAU 3}

\section{L'imitation de l'image, du mot et de la syntaxe morandiens entreprise par Liu Na'ou}

\begin{tabular}{|c|c|c|}
\hline $\begin{array}{l}\text { Citations dans La } \\
\text { nuit de six-jours }\end{array}$ & $\begin{array}{l}\text { Traduction chinoise de La } \\
\text { nuit des six-jours entreprise } \\
\text { par Dai Wangshu }\end{array}$ & $\begin{array}{l}\text { L'imitation de Morand dans l'écriture de Liu } \\
\text { Na'ou }\end{array}$ \\
\hline $\begin{array}{l}\text { Son beau teint juif } \\
\text { (Morand 1992: } \\
\text { 137) }\end{array}$ & $\begin{array}{l}\text { 美麗的猶太風的顏色 } \\
\text { (Muhang [Morand] 1929: } \\
\text { 54; traduction de Dai) }\end{array}$ & $\begin{array}{l}\text { 瘦小而隆直的希臘式的鼻子 } \\
\text { [Son nez mince, droit et grec] } \\
\text { 遊戲 [Le jeu }]^{41} \\
\text { (Liu 1997: } 3 \text {; notre traduction) }\end{array}$ \\
\hline $\begin{array}{l}\text { Trembler d'un } \\
\text { paludisme sacré } \\
\text { (Morand 1992: } \\
\text { 138) }\end{array}$ & $\begin{array}{l}\text { 像一種瘧疾的患者一樣地戰 } \\
\text { 慄著 } \\
\text { (Muhang 1929: } 55 ; \\
\text { traduction de Dai) }\end{array}$ & $\begin{array}{l}\text { 全身的筋肉也和著那癲癇性的節律, 發抖地戰慄 } \\
\text { 起來 } \\
\text { [Le muscle du corps frissonne et tremble en } \\
\text { rythme épileptique] } \\
\text { 遊戲 [Le jeu] (Liu } 1997: 2 \text {, notre traduction) }\end{array}$ \\
\hline $\begin{array}{l}\text { La salle sentait le } \\
\text { bouillon-minute, } \\
\text { l'œuf couvi, } \\
\text { l'aisselle et un Jour } \\
\text { viendra. } \\
\text { (Morand 1992: } \\
\text { 138) }\end{array}$ & $\begin{array}{l}\text { 廳中有肉汁的氣味, 孵退蛋 } \\
\text { 的氣味, 和Un jour viendra } \\
\text { 香水的氣味 } \\
\text { (Muhang 1929: 56; } \\
\text { traduction de Dai) }\end{array}$ & $\begin{array}{l}\text { No. 4711的香味, 白粉的, 襪子的, 汗汁的, 潮濕了 } \\
\text { 的皮包的, 脂油的, 酸化鐵的, 藥品的, 這些許多 } \\
\text { 的味混合起來造出一種氣味的cocktail } \\
\text { [La saveur de numéro } 4711 \text {, l'odeur de la cocaïne, } \\
\text { des chaussettes, de l'aisselle, du sac humide, de la } \\
\text { graisse, du fer acidifié, des médicaments, et } \\
\text { beaucoup de goût mélangé pour créer un gaz de } \\
\text { cocktail] } \\
\text { 風景 [Le paysage] (Liu 1997: 13, notre traduction) }\end{array}$ \\
\hline $\begin{array}{l}\text { Entre les tables les } \\
\text { sommeliers } \\
\text { volaient tenant } \\
\text { entre chaque doigt } \\
\text { un apéritif noir. } \\
\text { (Morand 1992: } \\
\text { 144) }\end{array}$ & $\begin{array}{l}\text { 侍者們在桌子間飛翔著, 在 } \\
\text { 每個手指間拿著一杯黑色的 } \\
\text { 飲料 } \\
\text { (Muhang 1929: 70; } \\
\text { traduction de Dai) }\end{array}$ & $\begin{array}{l}\text { 在這中間最精細又最敏捷的可算是那白衣的僕歐 } \\
\text { 的動作, 他們活潑潑地, 正像穿花的蛺蝶一樣, 由 } \\
\text { 這一邊飛到那一邊, 由那一邊又飛到別的一邊 } \\
\text { [Parmi eux, le mouvement des boys vêtus en blanc } \\
\text { est le plus délicat et agile, étant animés, ils sont } \\
\text { comme les papillons traversant des fleurs, volent } \\
\text { d'un côté à l'autre côté, et volent de cette côté-là à } \\
\text { une autre encore] } \\
\text { 遊戲 [Le jeu] (Liu 1997: 1, notre traduction) }\end{array}$ \\
\hline
\end{tabular}


Même si Liu Na'ou a d'abord lu les œuvres de Morand en traduction japonaise, et qu'il a relu les textes originaux après avoir appris le français en Chine, nous trouvons encore un lexique et une syntaxe qui ressemblent à la traduction chinoise de $\mathrm{La}$ nuit de six-jours. Par exemple, 《美麗的猶太風的顔色》 [son beau teint juif] (Morand 192: 137), 《瘦小而隆直的希臘式的鼻子» [son nez mince, droit et grec] (Liu Na'ou 1997: 3). Liu a décrit le portrait du personnage par des stéréotypes liés à son appartenance raciale. Cette démarche apparaît souvent dans les nouvelles de Morand; outre la citation de Morand, voici un autre exemple tiré de La nuit catalane (1922): «main britannique tachée de son; charnue main germanique» (Morand 1992: 77). Cet exemple montre aussi comment Morand esquisse des personnages à l'aide de connotations nationales. De plus, Morand a utilisé le mouvement du "paludisme» pour décrire le tremblement du joueur de jazz: 《像一種瘧疾的患者一樣地戰慄著》 [Trembler d'un paludisme sacré], tandis que Liu a employé le rythme de l'épileptique pour décrire le frisson des danseurs: 《全身的筋肉也和著那瘨瘉性的節律, 發抖地 戰慄起來” [Le muscle du corps frissonne et tremble en rythme épileptique]. C'est entièrement nouveau et exotique d'utiliser la maladie comme métaphore pour décrire le mouvement du corps dans la littérature chinoise. Sans mentionner que, dans le dernier exemple du Tableau 3, Morand a exagéré le mouvement des sommeliers par l'emploi du mot «voler», et ceci s'est bien intégré dans l'écriture de Liu.

Sur le plan des techniques littéraires, Morand aime composer des paragraphes comme un inventaire en énumérant des odeurs, des perceptions sensorielles variées dans une scène, afin de décrire l'espace urbain de façon minutieuse. Par exemple, «la salle sentait le bouillon-minute, l'œuf couvi, l'aisselle et un Jour viendra» (Morand 1992: 138); Morand énumère dans sa phrase les odeurs de la salle de danse. Liu emploie la même technique pour traduire ce passage dans 風景 [Le paysage]: «No. 4711 的香味, 白粉的, 襪子的, 汗汁的, 潮濕了的皮包的, 脂油的, 酸化鐵的, 藥品的, 這些許多的味混合起來造出一種氣味的 cocktail» [La saveur de numéro 4711, l'odeur de la cocaïne, des chaussettes, de l'aisselle, du sac humide, de la graisse, du fer acidifié, des médicaments, beaucoup de goût se mélangent pour créer un gaz de cocktail] (Liu Na'ou 1997: 13). Le texte traduit littéralement respecte la syntaxe du texte-source, il présente une juxtaposition de noms avec un seul verbe. L'écriture de Liu emprunte à Morand cette manière de décrire qui crée un espace matérialiste et érotique sur lequel le visage de la modernité urbaine éclate par l'inventaire d'odeurs d'objets kitch.

En vérité, les deux nouvelles de Liu citées ci-dessus, à savoir 遊戲 [Le jeu] et 風景 [Le paysage], proviennent de son premier et unique recueil de nouvelles都市風 景線 ([Scène], 1930) dans lequel il décrit de manière fragmentaire des éléments propres au paysage urbain de Shanghai tels que les femmes modernes, le jazz, la salle de bal, le boy de cafétéria, la rencontre sexuelle, etc. Aux yeux de Liu, pour décrire ces signifiants flottants de la ville qui sont très présents à Shanghai depuis la fin du $19^{\mathrm{e}}$ siècle et qui sont liés à la technologie moderne et à l'imitation du style de la vie occidentale, il faut utiliser des expressions suggérant la modernité. La lecture de textes européens traduits littéralement lui a fourni des exemples pour imiter la forme moderne. En réalité, Liu était loin d'être le premier à avoir essayé de repousser les limites de la langue chinoise qui ne possède pas d'équivalents pour traduire les néologismes occidentaux. Les poètes de la fin de la dynastie Qing avaient déjà commencé à réfléchir à la façon d'introduire les néologismes occidentaux au sein de la poésie 
classique. Les textes européens traduits littéralement ont constitué un grand pas en avant dans ce processus de modernisation de la langue chinoise, dans le sens où ils offrent une ressource au chinois pour absorber le vocabulaire, la syntaxe, la composition, les images des textes européens à grande échelle. C'est pourquoi le poète Zhu Ziqing (1898-1948) a écrit: «il faut absorber la langue vernaculaire des textes traduits pour favoriser à la fois la modernisation du chinois vernaculaire et la modernisation de la langue nationale ${ }^{42}$.»

\section{Conclusion}

Paul Morand est un écrivain prolifique sur lequel peu a été écrit avant les années 1980, que ce soit dans le champ de la critique ou de la biographie. Mieux connu en tant que nouvelliste, il a obtenu un grand succès lors de l'entre-deux-guerres, mais sa carrière a subi un revers suite à sa collaboration avec le gouvernement de Vichy. Même si ses œuvres ont connu un regain d'intérêt dans les années 1950, il n'a jamais regagné son ancienne popularité. Néanmoins, en ce qui concerne l'histoire des modernismes japonais et chinois, son nom est incontournable.

Pour se donner une chance de comprendre les enjeux véritables d'un texte diffusé à l'étranger, cet article a analysé la réception de Morand à partir d'une étape plus originelle, les traductions japonaise et chinoise de Morand en se concentrant sur deux nouvelles: La nuit romaine et La nuit des six-jours. Dans la traduction japonaise, en tant que poète, le traducteur Horiguchi a mis l'accent sur l'intensité sensorielle et poétique des nouvelles de Morand, il a renforcé le caractère descriptif des images afin de mettre en valeur le style métaphorique de Morand. Cela a contribué à ébranler la scène littéraire japonaise des années 1920, encore dominée par le naturalisme et sa variante locale le Shishōsetsu. Par ailleurs, Horiguchi a proposé d'aborder l'univers littéraire de Morand par le prisme de «la logique de la sensation». Dès lors, Morand fut invariablement associé au mot «sensation» et à l'École des sensations nouvelles dans les critiques et c'est à ce titre qu'il occupe une place essentielle dans le discours moderniste littéraire japonais.

Bien que l'introduction de Morand en Chine se soit faite à travers l'École des sensations nouvelles japonaise, la réception et la stratégie de traduction de Morand en Chine et au Japon sont différentes. Le traducteur chinois des nouvelles de Morand, Dai Wangshu, a adopté la démarche de la traduction littérale, un résultat qui confine à la "traduction raide», ce qui génère des problèmes: surabondance d'adjectifs, grammaire fautive, confusions sémantiques, etc. Mais cette stratégie de traduction fait écho à la construction d'une langue chinoise moderne vernaculaire dont l'une des approches est d'assimiler la syntaxe, la grammaire et le vocabulaire des langues européennes dans le chinois moderne à travers la traduction. En comparant les nouvelles de Liu Na'ou et la traduction chinoise de La nuit des six-jours de Morand, nous pouvons considérer que Liu a imité les images, le lexique et la syntaxe de Morand, et ceci montre que certains textes européens traduits littéralement ont contribué au processus de modernisation de la langue chinoise, dans le sens où ces traductions constituent autant de ressources permettant à la langue chinoise d'absorber à grande échelle le vocabulaire, la syntaxe, la composition et l'image des textes européens.

Comme cet article se limite au sujet «traduction dans le modernisme», certaines questions qui demeurent d'actualité ne sont pas évoquées ici. Par exemple, comment 
la traduction chinoise a-t-elle évolué sous l'influence des pratiques japonaises? Liu Na'ou a traduit «Paul Morand» de Benjamin Crémieux en chinois. Ce texte avait été traduit par Horiguchi dans sa préface de la version japonaise de Fermé la nuit. Par ailleurs, Dai Wangshu a traduit La nuit des six-jours en 1928, l'incluant dans 法蘭西 短篇傑作集 ([Chef-d'œuvre des nouvelles françaises]), puis l'a modifiée en 1929 dans 天女玉麗 [Céleste Julie]. Comparer les deux versions de cette dernière traduction permet de constater que toutes deux ont également repris les kanjis utilisés dans la traduction japonaise. Nous avons étudié les kanjis 《冬期自由車競爭場》 [Vel'd'hiv'], 《蜂組»[Les Guêpes] et 《柘榴水» [Grenadine] à titre d'exemples:

\section{TABLeAu 4}

\section{Les kanjis utilisés dans les deux versions de la traduction chinoise de Dai Wangshu}

\begin{tabular}{|c|c|c|}
\hline $\begin{array}{l}\text { Deux versions de la traduction chinoise de } \\
\text { Dai Wangshu }\end{array}$ & $\begin{array}{l}\text { Traduction japonaise } \\
\text { d'Horiguchi Daigaku }\end{array}$ & Texte original français \\
\hline $\begin{array}{l}\text { Chef-d'euvre des nouvelles françaises (la } \\
\text { première version de la traduction) } \\
\text { 我要到冬期自由車競爭場去看兩點鐘的賞金 } \\
\text { 競技。 } \\
\text { (Muhang [Morand] 1928: 6, traduction de } \\
\text { Dai) } \\
\text { Céleste Julie (la deuxième version de la } \\
\text { traduction) } \\
\text { 我要到冬期自由車競爭場去看兩點鐘的賞金 } \\
\text { 競技。(Muhang 1929: 58, traduction de Dai) }\end{array}$ & $\begin{array}{l}\text { 妾はこれから二時の賞金競 } \\
\text { 爭を見る為めに冬期自轉車競 } \\
\text { 爭場 へ行くのです。 } \\
\text { (Horiguchi 1986: 284) }\end{array}$ & $\begin{array}{l}\text { Je vais au Vel'd'hiv' } \\
\text { pour les primes de } 2 \\
\text { heures. } \\
\text { (Morand 1992: 139) }\end{array}$ \\
\hline $\begin{array}{l}\text { Chef-d'ouvre des nouvelles françaises: } \\
\text { 黃色的和黑色的 }[\cdots] \text { 『蜂組』 }[\cdots] \text { 么牌 } \\
\text { 隊。 } \\
\text { (Muhang 1928: 6, traduction de Dai) } \\
\text { Céleste Julie: } \\
\text { 黃色的和黑色的 [...]『蜂組』 [...] 么牌 } \\
\text { 隊。 } \\
\text { (Muhang 1929: 59, traduction de Dai) }\end{array}$ & $\begin{array}{l}{[\cdots \cdots] \text { 黃色と黑の縞のシャツ }} \\
{[\cdots] \text { あれが『蜂組』です。 }} \\
\text { (Horiguchi 1986:284) }\end{array}$ & $\begin{array}{l}\text { Jaune et noir }[\ldots] \text { Les } \\
\text { Guêpes [...] l'équipe } \\
\text { des as. } \\
\text { (Morand 1992: 139) }\end{array}$ \\
\hline $\begin{array}{l}\text { Chef-d'ouvre des nouvelles françaises: } \\
\text { 落日, 柘榴水。時間是像地瀝青一樣地平 } \\
\text { 滑。(Muhang 1928: 15, traduction de Dai) } \\
\text { Céleste Julie: } \\
\text { 落日, 柘榴水。時間是像地瀝青一樣平滑。 } \\
\text { (Muhang 1929: 68, traduction de Dai) }\end{array}$ & $\begin{array}{l}\text { 夕日の光。柘榴水。アスフアル } \\
\text { トのやうに時は滑かに流れ } \\
\text { た。 } \\
\text { (Horiguchi } 1986: 288)\end{array}$ & $\begin{array}{l}\text { Coucher de soleil: } \\
\text { Grenadine. L'heure } \\
\text { était facile comme } \\
\text { l'asphalte. } \\
\text { (Morand 1992: 144) }\end{array}$ \\
\hline
\end{tabular}

D’ailleurs, dans certains de cas, Dai Wangshu a, de toute évidence, traduit le texte d'après le texte original français; et ce, de manière tout à fait correcte. Dai Wangshu a cependant effectué des modifications dans la deuxième version de sa traduction, essentiellement en réaction à la traduction japonaise de La nuit des sixjours (1924). Cette seconde version, qui fait référence à la traduction japonaise, n'est pas aussi correcte que la première: la traduction japonaise a, en quelque sorte, faussé la seconde version de la traduction de Dai Wangshu. Voici un exemple: 
TABLEAU 5

La modification de la deuxième version de la traduction de Dai

\begin{tabular}{|c|c|c|}
\hline $\begin{array}{l}\text { Deux versions de la traduction chinoise de La } \\
\text { nuit des six-jours entreprises par Dai Wangshu }\end{array}$ & $\begin{array}{l}\text { La traduction japonaise } \\
\text { d'Horiguchi Daigaku }\end{array}$ & Le texte original français \\
\hline $\begin{array}{l}\text { Chef-d'euvre des nouvelles françaises: } \\
\text { 在帶著一種神聖的瘧疾戰慄著。(Muhang 1928: } \\
\text { 3, traduction de Dai) } \\
\text { Céleste Julie: } \\
\text { 像一種瘧疾的患者一樣地戰慄著。 } \\
\text { (Muhang 1929: } 55 \text {, traduction de Dai) }\end{array}$ & $\begin{array}{l}\text { マラリヤ患者のやうに身 } \\
\text { を震はせ[...] } \\
\text { (Horiguchi 1986: 282) }\end{array}$ & $\begin{array}{l}{[\ldots] \text { trembler d'un }} \\
\text { paludisme sacré. } \\
\text { (Morand 1992: 137) }\end{array}$ \\
\hline
\end{tabular}

Dans la première version, Dai Wangshu a traduit la phrase «trembler d'un paludisme sacré » correctement en 《在帶著一種神聖的瘧疾戰慄著》(Dai 1928: 3), mais dans la deuxième version, il a repris la traduction japonaise «マラリヤ患者のやうに 身を震はせ» (Horiguchi 1986: 282) en supprimant l'adjectif «sacré»[神聖的] (Morand 1992: 137). La deuxième version devient donc: 《像一種瘧疾的患者一樣地 戰慄著》 [trembler d'un paludisme]. Dans ce dernier cas, Dai Wangshu a fait une omission de traduction. Cet exemple fait partie des passages qui prouvent que Dai Wangshu a repris la traduction japonaise de La nuit des six-jours. Dans la plupart de cas, Dai Wangshu a littéralement traduit les œuvres de Morand, dans certain de cas, il a imité la traduction japonaise du même texte. Cette attitude paraît contradictoire et l'on peut s'interroger sur les raisons qui ont conduit Dai Wangshu à modifier sa démarche, ainsi que sur les autres textes japonais que Dai Wangshu a pu mobiliser. Ces questions, trop complexes pour être traitées dans le cadre de cet article, feront l'objet d'une autre étude de notre part, actuellement en cours, qui a notamment pour objet d'analyser les relations intertextuelles entre la traduction japonaise et le texte original de Morand au sein même de la traduction chinoise.

\section{NOTES}

1. L'École des sensations nouvelles est un courant littéraire moderniste de l'ère Taishō (1912-1926). Elle est étroitement liée à la revue 文藝時代 [L'Époque de la littérature] parce que les membres de l'École sont en fait les écrivains clés de la revue. Les plus connus sont Yokomitsu Riichi (1898-1947), Kawabata Yasunari (1899-1972), Tōkō Kon (1898-1977) et Nakagawa Yoichi (1897-1994). Dans le numéro inaugural de la revue (1924), Yokomitsu Riichi publie une nouvelle 頭ならびに腹 [La tête et le ventre] qui a une certaine tonalité symboliste et qui exprime un esprit de révolte à l'encontre de la littérature naturaliste.

2. Texte original: 新感覚派論争の動態を分析し、その文学史的意義を檢討することは、問題視角の いかんを問わず、日本におけるモダニズム文学の全体像を、特質と限界に統一的に解明するため の、必要欠くべからざる前提作業であるのみならず $[\cdots]$. La traduction française de ce texte est réalisée par l'auteur de cet article. Si l'auteur de cet article n'indique pas la source des traductions dans les notes suivantes, cela signifie que celles-ci ont aussi été réalisées par elle.

3. Ouvert la nuit est le deuxième recueil de nouvelles de Morand. Le recueil a obtenu un grand succès en France. Il comporte six nouvelles, à savoir "La nuit catalane», "La nuit turque», "La nuit romaine», «La nuit des six-jours», "La nuit hongroise» et «La nuit nordique». MorAnD, Paul (1922/1992): Ouvert la nuit. In: Nouvelles complètes. (Éd. par Michel Collomb) Tome 1. Paris: Gallimard-Pléiade, 68-174. Les dates de la première publication des œuvres et des articles souvent citées à plusieurs reprises dans le texte seront indiquées dans les notes et la bibliographie.

4. Сніва, Kameo (1924/1956-1957): Shinkankakuha no tanjō [La naissance de l'École des sensations nouvelles]. In: Ken Hirano, Hideo Odagiri et Kenkichi Yamamoto, dir.: Gendai Nihon bungaku ronsōshi [Histoire des débats de la littérature moderne japonaise]. Vol. 1. Tokyo: Miraisha, 193196. 
5. Dans le journal de Liu Na’ou, nous remarquons qu'à partir de 1927, il a déjà lu la traduction japonaise des œuvres de Morand et amorcé la traduction des œuvres de l'École des sensations nouvelles japonaise en langue chinoise. Le 29 avril, il précise avoir lu «Mr.U» de Morand; le 18 octobre, il a lu plusieurs poèmes de Morand; le 23 octobre, il a fini la lecture d'un recueil de poèmes de Morand et fait une remarque: "J'ai fini la lecture du recueil de poèmes de Morand ce soir, les mots sont difficiles, bruts et inaccomplis, bien que la tonalité moderniste soit très forte, elle est superficielle: il y a seulement quelques mots qui reflètent la sensation nouvelle.» (Texte original: 晚上把モラン 詩集唸完, 難字多, 粗而不滑, 雖然現代色很濃, 可是並不深, 只多了幾個新感覺的字。) Voir KANG, Laixin et Xu, Qinzhen éd. (2001): Liu Na'ou quanji: Rijiji [CEuvres complètes de Liu Na'ou: Le Journal]. (Traduit du chinois par Hsiao-yen Peng et Yingzhe Huang) Tainanxian Xinyingshi: Tainanxian wenhuaju, 652, 658, 662 .

6. Edward Bulwer-Lytton est un homme politique, poète et romancier britannique du $19^{\mathrm{e}}$ siècle. Son œuvre Ernest Maltravers (1837) a été traduite en japonais par Niwa Jun'ichirō (1851-1919) en 1879, intitulée 花柳春話: 欧州奇事 [Karyū shunwa: Ōshū kiji]. Fujita Mokichi (1852-1892) et Ozaki Yasuo (date inconnue) ont traduit une autre de ses œuvres Kenelm Chillingly: His Adventures and Opinions (1873) en 1885, intitulée 慗思談：諷世嘲俗 [Kenshidan: Fūsei chōzoku].

7. Benjamin Disraeli, homme politique et auteur britannique, nommé deux fois premier ministre du Royaume-Uni. Son œuvre Coningsby, or the New Generation (1844) a été traduite en japonais par Seki Naohiko (1857-1934) en 1884, intitulée 春鶯囀：政党余談 [Shun'ōden: Seitō yodan].

8. Jules Verne est un écrivain français. Son œuvre Le tour du monde en quatre-vingts jours (1873), intitulée 八十日間世界一周 [Hachijūnichikan sekai isshū] en japonais, a été traduite par Kawashima Chūnosuke (1853-1938) en 1878. Elle était la première œuvre de science-fiction traduite au Japon. L'autre œuvre de Verne, De la terre à la Lune (1865), a été traduite par Iwaya Sazanami (1870-1933) et Inoue Tsutomu (1850-1928) en 1880.

9. Le terme 硬譯 [la traduction raide] a été inventé par Lu Xun (1881-1936), qui est en vérité une manière de traduction littérale. Quand Lu Xun faisait la traduction pendant les années 1920 et 1930, il a changé sa manière de traduction à une approche de mot-à-mot. Cette approche de traduction a été appliquée surtout à sa traduction de la littérature russe, des œuvres marxistes et léninistes. Lu, Xun (1930/1956): «Yingyi» yu wenxue de jiejixing [ "La traduction raide» et la classe de la littérature]. In: Lu Xun quanji [Euvres complètes de Lu Xun]. Beijing: Renmin wenxue chubanshe, 155-173.

10. Il existe de nombreuses recherches sur les modernismes des littératures japonaise et chinoise, par exemple, pour le modernisme japonais: CHiBA, Sen'ichi (1978): Gendai bungaku no hikaku bungaku teki kenkyū: Modānizumu no shi teki dōtai [Les Études comparatistes de la littérature moderne: l'histoire du modernisme]. Tokyo: Yagi shoten.; Tyler, William J., dir. (2008): Modanizumu: Modernist Fiction from Japan 1913-1938. Honolulu: University of Hawaii Press.; STARRs, Roy éd. (2012): Rethinking Japanese Modernism. Leiden et Boston: Global Oriental. Pour le modernisme chinois: YAN, Jiayan (1985): Qianyan [Introduction]. In: YAN, Jiayan, dir. : Xinganjuepai xiaoshuo xuan [Sélection de l'École des sensations nouvelles]. Beijing: Renmin wenxue chubanshe, 1-38.; LEE, Ou-fan (2001): Shanghai modeng: yizhong xin dushi wenhua zai Zhongguo 1930-1945 [Shanghai Modern: The Flowering of a New Urban Culture in China, 1930-1945 (le titre original)]. (Traduit par Jian MAO) Beijing: Beijing daxue chubanshe.

11. Seulement la première moitié de La nuit nordique a été traduite parce que, selon Horiguchi, la seconde moitié de la nouvelle au cours de laquelle le narrateur a une relation sexuelle avec une femme finlandaise aurait pu susciter des controverses ou même aurait pu être interdite de publication. Cité de Tsuchiya, Satoshi (2006): Taishō ki ni okeru Horiguchi Daigaku no hon'yaku [La traduction d'Horiguchi Daigaku dans l'ère Taishō]. In: Nihon kindai bungaku-kai henshū iinkai éd.: Nihon kindai bungaku [La littérature moderne japonaise]. Vol. 75, 99.

12. À l'âge de 19 ans (1911), Horiguchi se rend au Mexique où son père est diplomate. Il visite également la Belgique, l'Espagne, la Suisse, la France, le Brésil et la Roumanie à l'occasion des mutations successives de son père. Il rentre au Japon en 1925, au terme d'un séjour à l'étranger de 14 ans.

13. Lors de ses brefs séjours en Espagne, à Paris, au Brésil et en Roumanie, Horiguchi a entretenu une correspondance avec Marie Laurencin (1883-1956) et Thomas Mann (1875-1955).

14. Texte original: 堀口君の佛蘭西語と日本語とは、十分信賴してい […]. IkutA, Chōkō (1925/19561957): Bundan no shinjidai ni ataeu [Remarques sur une nouvelle époque de la communauté littéraire]. In: Ken Hirano, Hideo Odagiri et Kenkichi Yamamoto, dir.: Gendai Nihon bungaku ronsōshi [Histoire des débats de la littérature moderne japonaise]. Vol. 1. Tokyo: Miraisha, 215. 
15. 月光とピエロ [Le clair de lune et Pierrot], premier recueil de poèmes d'Horiguchi Daigaku, qui a été préfacé par Nagai Kafū (1879-1959). Les poèmes du recueil décrivent l'amour et la mélancolie du jeune homme (Horiguchi avait 27 ans lorsqu'il a publié ce recueil). Le recueil est dans un style symboliste, entremêlé de mots français. Horiguchi, Daigaku (1919): Gekko to Pierrot [Le clair de lune et Pierrot]. Tokyo: Momiyama shoten.

16. Texte original: ポオル・モオランの文章は人を驚かせる。何故であるか?理由は至極簡単である。 それは鋭敏な感受性と観察力を持ったこの新文章家が事物を在来の文章の中にはかつて試みら れなかった新しい關係によって結びつけるからである。在来の文章の中にあっては、事物の関係 はすべて「理性の論理」で結びつけられていたのである。然るにポオル・モオランは「理性の論 理」に代えるに「感覚の論理」を以てした。HoRIGUCHI, Daigaku (1924/1986): Horiguchi Daigaku zenshū [Euvres complètes d'Horiguchi Daigaku]. Vol. 8. Tokyo: Ozawa shoten, 220.

17. L'Homme pressé est un roman de Paul Morand paru en 1941. Il évoque avec plein d'humour et dans une langue très riche un sujet éternel: le temps. MorAnd, Paul (1941/1990): L'Homme pressé. Paris: Gallimard DL.

18. Dans 現代日本文学論争史 [Histoire des débats de la littérature moderne japonaise] (1956-1957), dix articles sont consacrés au débat autour de l'École des sensations nouvelles, parmi lesquels quatre mentionnent Morand: 新感覺派の誕生 [La naissance de l'École des sensations nouvelles] de CHIBA Kameo; 文壇の新時代に與う [Remarques sur une nouvelle époque de la communauté littéraire] d'IKuta Chōkō; 序にもう少し新しく [Préface: plus de la nouveauté] d'IKuTa Chōkō; 生田長江氏 に酬ゆその他(抄) [Réponse à Ikuta Chōkō et les autres (extrait)] d’ITō Einosuke. Ces quatre articles se concentrent sur le style ou les techniques littéraires d'Ouvert la nuit (voir note 3 pour la référence), à l'exception de 文壇の新時代に與う [Remarques sur une nouvelle époque de la communauté littéraire] d'Ikuta Chōkō qui évoque aussi la crise et la décadence des années 1920 décrites dans les nouvelles de Morand.

19. À ce sujet, voir ŌMura, Azusa (2011): The Paradox of Modernism: Paul Morand and the Literature of Japanese Modernism. Thèse de doctorat. Tokyo: Industrial University of Tokyo, 94-102.

20. Proust, Marcel (1921/1992): Préface à Tendre stocks. In: Nouvelles complètes. (Éd. par Michel Соцlомв) Tome 1. Paris: Gallimard-Pléiade, 11.

21. À la fin du $19^{\mathrm{e}}$ siècle et au début du $20^{\mathrm{e}}$ siècle, le concept de naturalisme japonais est très près de la conception de la vérité du naturalisme français: des expressions comme «la description plate», «la description explicite» témoignent de leur volonté de poursuivre cette conception. Néanmoins, aux yeux des naturalistes japonais, le but de «la vérité » est d'exprimer ou de présenter le soi, les sentiments du soi, la vie du soi. L'écrivain fait lui-même partie de son œuvre parce que dans sa logique, rien n'est plus vrai que les événements et les sentiments vus et vécus. Dans cette interprétation de «la vérité», l'écriture est alors une expérience de l'autoportrait et l'auteur devient le propre héros de son roman. Le Shishōsetsu [私小説], centré sur le récit de la vie privée de l'écrivain, est ainsi né. Soi-disant un récit de fiction, il est souvent considéré comme un journal intime.

22. Texte original: 自然は自然である、善でも無い、悪でも無い、美でも無い、醜でも無い $[\cdots]$ 。 Kosugr, Tengai (1901/1967): Hatsusugata [La parure du Nouvel An]. In: Kosugi Tengai, Oguri Fūyō, Okamoto Kidō, Mayama Seika shū [Collection de Kosugi Tengai, Oguri Fūyō, Okamoto Kidō et Mayama Seika]. Tokyo: Chikuma shobō, 63.

23. Texte original: だ゙見たま〉聴いたま〉触れたま〉の現実をさながらに描く。TAYAMA, Katai (1911/1977): Byōsha ron [Sur la description]. In: Tayama Katai shū [Collection de Tayama Katai]. Tokyo: Chikuma shobō, 393.

24. Texte original: それには屋外で起こった事を一切ぬきにして、すべてを屋内の光景にのみ限ろうと した。台所から書き、立関から書き、庭から書きして見た。川の音の聞こえる部屋まで行って、はじ めてその川のことを書いてみた。ShimaZaKi, Tōson (1937/1985): Ori ni furete [De temps en temps]. In: Shimazaki Tōson senshū [Ëuvres choisies de Shimazaki Tōson]. Vol. 11. Tokyo: Chikuma shobō, 234.

25. «La nuit de Portofino Kulm» est la nouvelle inaugurale du recueil Fermé la nuit (1923) de Paul Morand. Morand, Paul (1923/1992): La nuit de Portofino Kulm. In: Nouvelles complètes. (Éd. par Michel Cоццомв) Tome 1. Paris: Gallimard-Pléiade, 177-215.

26. Texte original: われ等は假りにそれを名づけて、「新感覺時代」だといひたい。それは、いかに好 んで特殊な視界の絶冝に立って、その視野の中から、いかに隱れた人生の大全面を透射し、 $[\cdots]$ 彼等が人生を表現するに、わざわざ『小さな穴』を撰ばねばならぬかといふならば $[\cdots]$ 微妙な 態度の芸術が発生するものも自然の約束なのである。さらば、なぜ、彼らが大きな内部人生を象徵 させるために使った、その小さな外形は、有りやうは、彼等が端的に刺激された、刹那の感覚の點 出に過ぎないからである。Voir note 4. 
27. Voir note 5 .

28. Crémieux, Benjamin (1924/2010): Paul Morand. In: Benjamin Crémieux, XXe siècle (Textes établis, préfacés et annotés par Catherine Helbert) Paris: Gallimard, 213-223.

29. Avant que Liu Na'ou n'ait officiellement introduit Morand dans les cercles modernistes de Shanghai, une première traduction des œuvres de Morand était déjà parue en septembre 1921 dans la revue littéraire chinoise 日晏 [Le soleil], à partir d'une traduction anglaise de La nuit turque (1922), mais elle ne suscita aucun écho dans les milieux littéraires chinois. Cité de LEe, Ou-fan (2001): Shanghai modeng: yizhong xin dushi wenhua zai Zhongguo 1930-1945 [Shanghai Modern: The Flowering of a New Urban Culture in China, 1930-1945 (le titre original)], (traduit par Jian MAo). Beijing: Beijing daxue chubanshe, 245.

30. Dans 戰爭語境下現代主義的反思一一保爾穆杭《六日之夜》從1928到1956年的四種中文翻譯 [La réflexion du modernisme dans le contexte de la guerre - quatre versions de la traduction chinoise de La nuit des six-jours entre 1928 et 1956], Kwong Hoyee a souligné que Dai Wangshu avait modifié la traduction de La nuit des six-jours à quatre reprises: en 1928, 1929, 1945 et 1956 (Voir Kwong, Hoyee (2014): Zhanzheng yuji xia xiandai zhuyi de fanxi-Bao'er Muhang Liuri zhi ye cong 1928 dao 1956 nian de sizhong zhongwen fanyi [La réflexion du modernisme sous le contexte de la guerre - quatre versions de la traduction chinoise de La nuit des six-jours entre 1928 et 1956]. In: Zhongguo xiandai wenxue yanjiu congkan [La série des recherches de la littérature modern chinoise]. Vol.10, 128-139). La première version de la traduction de La nuit des six-jours a été inclue dans 法蘭西短篇傑作集(第一集) [Chef-d'œuvre des nouvelles françaises] (vol. 1). Muhang [Morand] (1922/1928): Liuri zhiye [La nuit des six-jours]. In: Falanxi duanpian jiezuoji (di yi ji) [Chef-d'cuvre des nouvelles françaises], (traduit par Wangshu DAI). Vol. 1. Shanghai: Shuimo chubanshe, 1-25.

31. Charles d'Orléans est un prince français et un poète remarquable du XV $\mathrm{X}^{\mathrm{e}}$ siècle. Il est connu surtout pour ses œuvres poétiques écrites lors de sa longue captivité anglaise. Le printemps, parfois titré Rondeau, est une ode et les vers sont écrits en octets au lieu d'en alexandrins ou en dizain. D’orléans, Charles (1923-1924) : Poésies (Éd. par Pierre Champion). Vol. 2. Paris: H. Champion.

32. Au printemps de 1926, Dai Wangshu et ses deux camarades du cours de français à l'Université d'Aurore, Du Heng (1907-1964) et Shi Zhecun (1905-2003), ont publié leur première revue de coterie 嬰珞 [Collier de jade] qui était une revue trimensuelle et avait seulement 16 pages. le contenu était essentiellement composé par les poèmes, les essais et les textes traduits produits par ces trois fondateurs de la revue. Mais la revue n’a duré que quatre numéros et ses travaux de création ne leur ont fait accéder à aucune renommée dans la communauté littéraire shanghaienne à l'époque.

33. La dame aux camélias est un roman d'Alexandre Dumas fils, il est publié pour la première fois à Paris, en 1848, chez Cadot.

34. Par exemple, ce passage de Dai sur son voyage en Espagne: 和那吹著冰雪的風, 飄著憂鬱的雲的 俄羅斯比起來, 西班牙的土地是更饒於詩情一點。在那裡, 一切都邀人入夢, 催人懷古: 一溪一 石、一樹一花、山頭碉堡、風際牛羊 [… [Et le vent qui souffle de la neige, Comparé à la Russie où le vent charrie des flocons de neige et où flottent des nuages mélancoliques, la terre de l'Espagne est plus poétique. Là-bas, tout invite à rêver, tout invite à la nostalgie: une rivière ou une pierre, un arbre ou une fleur, un fort dans la montagne, les bovins et les moutons qui traversent le vent...] (traduction de l'auteur de l'article) DAI, Wangshu (1993): Dai Wangshu 1905-1950. (Éd. par Zhecun Shi et Guojing YING) Beijing: Renmin wenxue chubanshe, 150.

35. Le Mouvement du chinois vernaculaire ou le Mouvement du baihua (白話文運動, 1919) est mouvement qui préconise du remplacement du wenyan [文言] au baihua [白話]. Le wenyan (langue classique chinoise) est l'apanage d'une élite et cessa d'être la référence millénaire officielle de l'écrit à partir de 1917 à l'instigation d'intellectuels et d'hommes de lettres, tel Hu Shi (1891-1962), dans le cadre du grand mouvement anti-impérialiste et anti-confucéen qui est le Mouvement du Qautre Mai 1919. L'adoption du baihua (chinois vernaculaire), qui pourrait apparaître comme une simple décision technique, constitue en réalité une rupture fondamentale pour tous ceux qui recherchent à cette époque auprès de l'Occident les remèdes permettant à la Chine de s'engager sur la voie de la modernisation. Billion, Roger (1994): De la notion de modernité en chinois moderne: champs sémantiques et applications. In: Yves VADÉ, dir.: Ce que modernité veut dire. Tome 1. Bordeaux: Presses universitaires de Bordeaux, 99-100.

36. Le Huaben (話本) est un genre de conte qui est né sous la dynastie Song (960-1279). Il raconte des contes, des histoires merveilleuses ou étranges en langue vernaculaire de l'époque. 
37. 水澈傳 [Au bord de l'eau], littéralement «Le Récit des berges», est un roman d'aventures tiré de la tradition orale chinoise, compilé et écrit par plusieurs auteurs, mais en général attribué à Shi Nai'an (1296-environ1370). Il relate les exploits de cent huit bandits, révoltés contre la corruption du gouvernement et des hauts fonctionnaires de la cour de l'empereur de la dynastie Song. Le roman est considéré comme l'un des quatre grands romans classiques de la dynastie Ming (13681644) et est écrit en chinois vernaculaire de l'époque.

38. 西遊記 [La pérégrination vers l'Ouest] est un roman de Wu Cheng'en (1500-1582). Il fait partie des quatre grands romans classiques et comme 水澈傳 [Au bord de l'eau], il est aussi écrit en chinois vernaculaire de l'époque. Le roman retrace l'expédition du moine bouddhiste Xuanzang et de ses quatre protégeurs.

39. Texte original: 直用西洋文的款式、文法、詞法、章法、詞枝 $(\cdots \cdots \cdots)$ 一切修辭上的方法, 造成一種 超於現在的國語, 歐化的國語, 因而成就一種歐化國語的文學。Fu, Sinian (1919/1935): Zenyang zuo baihuawen [Comment faire l'écriture du baihua]. In: Jiabi Zhao éd.: Zhongguo xinwenxue daixi: jianshe lilunji [Nouvelle littérature chinoise: la construction de la théorie]. Shanghai: Shanghai liangyou tushu gongsi, 223.

40. Texte original: 白話文不但不全跟著國語的口語走, 也不全跟著傳統的白話走, 卻有意的跟著翻 譯的白話走。這是白話文的現代化, 也就是國語的現代化。[Il faut que le chinois vernaculaire n'adopte pas seulement la langue parlée, ni le chinois vernaculaire ancien, mais aussi la langue vernaculaire de la traduction. C'est la modernisation du chinois vernaculaire, c'est-à-dire la modernisation de la langue nationale. (Traduction de l'auteur de l'article)] ZHU, Ziqing: Baihua yu wenyan. In: Qiaosen ZHu éd.: Zhu Ziqing quanji [CEuvres complètes de Zhu Ziqing]. Vol. 8. Nanjing: Jiangsu jiaoyu chubanshe, 200.

41. Les deux nouvelles de Liu Na’ou citées ici, à savoir 遊戲 [Le jeu] et 風景 [Le paysage], proviennent du premier et unique recueil de nouvelles de Liu, 都市風景線 [Scène] (1930). LiU, Na’ou (1930/1997): Youxi [Le jeu], Fengjing [Le paysage]. In: Liu Na'ou xiaoshuo quanpian [Nouvelles complètes de Liu Na'ou]. Shanghai: Xuelin chubanshe, 1-8, 9-15.

42. Zhu, Ziqing (1935/1988): Baihua yu wenyan. In: Qiaosen Zhu, dir.: Zhu Ziqing quanji [CEuvres complètes de Zhu Ziqing]. Vol. 8. Nanjing: Jiangsu Jiaoyu Chubanshe, 200.

\section{RÉFÉRENCES}

Amoia, Alba F. et KnApp, Bettina L. (2002): Multicultural Writers from Antiquity to 1945: A Bio-Bibliographical Sourcebook. Westport: Greenwood Press, 183-186.

Billion, Roger (1994): De la notion de modernité en chinois moderne: champs sémantiques et applications. In: Yves VADÉ, dir.: Ce que modernité veut dire. Tome 1. Bordeaux: Presses universitaires de Bordeaux, 99-108.

ChibA, Kameo (1924/1956-1957): 新感覚派の誕生 (Shinkankakuha no tanjō) [La naissance de l’École des sensations nouvelles]. In: Ken Hirano, Hideo Odagiri et Kenkichi Yamamoto, dir.: 現代日本文学論争史 (Gendai Nihon bungaku ronsōshi) [Histoire des débats de la littérature moderne japonaise]. Vol. 1. Tokyo: Miraisha, 193-201.

CHIBA, Sen'ichi (1978): 現代文学の比較文学的研究: モダーニズムの史的動態 (Gendai bungaku no hikaku bungaku teki kenkyū: Modānizumu no shi teki dōtai) [Les études comparatistes de la littérature moderne: l'histoire du modernisme]. Tokyo: Yagi Shoten.

Cogny, Pierre (1976): Le Naturalisme. Paris: PUF.

Crémieux, Benjamin (1924/2010): Paul Morand. In: Benjamin Crémieux, XXe siècle (Textes établis, préfacés et annotés par Catherine Helbert) Paris: Gallimard, 213-223.

DAI, Wangshu (1993): 戴望舒 1905-1950 [Dai Wangshu 1905-1950]. (Éd. par Zhecun SHI et Guojing YING) Beijing: Renmin wenxue chubanshe.

D’orléans, Charles (1923-1924) : Poésies. (Éd. par Pierre Champion) Vol. 2. Paris: H. Champion. Douzou, Catherine (2003): Paul Morand nouvelliste. Paris: H. Champion.

Fu, Sinian, (1919/1935): 怎樣做白話文 (Zenyang zuo baihuawen) [Comment faire l'écriture du baihua]. In: Jiabi Z HAO, éd.: 中國新文學大系: 建設理論集 (Zhongguo xinwenxue daixi: jianshe lilunji) [Nouvelle littérature chinoise: la construction de la théorie]. Shanghai: Shanghai liangyou tushu gongsi, 217-227.

Guo, Yanli (1997): 中國近代翻譯文學概論 (Zhongguo jindai fanyi wenxue gailun) [Traduction de la littérature moderne en Chine: l'introduction]. Hubei: Hubei jiaoyu chubanshe. 
Horiguchi, Daigaku (1986): Horiguchi Daigaku zenshū [Euvres complètes d'Horiguchi Daigaku]. Vol. 8. Tokyo: Ozawa shoten.

HoRIGUCHI, Daigaku (1925): 月下の一群 (Gekka no ichigun) [Un troupeau au clair de lune]. Tokyo: Daiichi shobō.

Horiguchi, Daigaku (1919): 月光とピエロ (Gekko to Pierrot) [Le clair de lune et Pierrot]. Tokyo : Momiyama shoten.

Horiguchi, Daigaku (1926): 砂の枕 (Suna no makura) [L'oreiller de sable]. Tokyo: Daiichi shobō.

IKUTA, Chōkō (1925/1956-1957): 文壇の新時代に與え (Bundan no shinjidai ni ataeu) [Remarques sur une nouvelle époque de la communauté littéraire]. In: Ken Hirano, Hideo Odagiri et Kenkichi Yамамото, dir.: 現代日本文学論争史 (Gendai Nihon bungaku ronsōshi) [Histoire des débats de la littérature moderne japonaise]. Vol. 1. Tokyo: Miraisha, 214-225.

IKuTA, Chōkō (1925/1956-1957)：序にもう少し新しく [Jo ni mōsukoshi atarashiku] [Préface: plus de la nouveauté]. In: Ken Hirano, Hideo Odagiri et Kenkichi Yамamoto, dir.: 現 代日本文学論争史 (Gendai Nihon bungaku ronsōshi) [Histoire des débats de la littérature moderne japonaise]. Vol. 1. Tokyo: Miraisha, 231-239.

ITō, Einosuke (1925/1956-1957): 生田長江氏に酬ゆその他(抄) (Ikuta Chōkō shi ni mukuyu sonota (shou)) [Réponse à Ikuta Chōkō et les autres (extrait)]. In: Ken Hirano, Hideo Odagiri et Kenkichi Yамамото, dir. (1956-1957): 現代日本文学論争史 (Gendai Nihon bungaku ronsōshi) [Histoire des débats de la littérature moderne japonaise]. Vol. 1. Tokyo: Miraisha, 239-241.

KANG, Laixin et Xu, Qinzhen, dir. (2001): Liu Na'ou quanji: Rijiji [Euvres complètes de Liu Na’ou: Le Journal], (traduit du chinois par Hsiaoyen Peng et Yingzhe Hunng). Tainanxian Xinyingshi: Tainanxian wenhuaju.

Kosugi, Tengai, Oguri, Fūyō, Oкамото, Kidō et al. (1967): 小杉天外・小栗風葉・岡本綺堂・ 眞山青果集 (Kosugi Tengai, Oguri Fūyō, Okamoto Kidō, Mayama Seika shū) [Collection de Kosugi Tengai, Oguri Fūyō, Okamoto Kidō et Mayama Seika]. Tōkyō: Chikuma Shobō.

KwONG, Hoyee (2014): 戰爭語境下現代主義的反思一一保爾・穆杭〈六日之夜〉從 1928 到 1956 年的四種中文翻譯 (Zhanzheng yuji xia xiandai zhuyi de fanxi - Bao’er Muhang Liuri zhi ye cong 1928 dao 1956 nian de sizhong zhongwen fanyi) [La réflexion du modernisme sous le contexte de la guerre - quatre versions de la traduction chinoise de La nuit des six-jours entre 1928 et 1956]. In: Zhongguo xiandai wenxue yanjiu congkan [La série des recherches de la littérature modern chinoise]. 10: 128-139.

LeE, Gregory (1989): Dai Wangshu: The Life and Poetry of a Chinese Modernist, Hong Kong: Chinese University Press.

LEE , Ou-fan (2001): 上海摩登———種都市文化在中國 1930-1945 (Shanghai modeng-yizhong dushi wenhua zai Zhongguo 1930-1945) [Shanghai Modern: The Flowering of a New Urban Culture in China, 1930-1945], (traduit par Jian MAO). Beijing: Beijing daxue chubanshe.

Liu, Na'ou (1997): 劉呐鷗小說全篇 (Liu Na’ou xiaoshuo quanpian) [Nouvelles de Liu Na'ou]. Shanghai: Xuelin chubanshe.

LiU, Na'ou, DAI, Wangshu, ShI, Zhecun, et al. (1928): 無軌列車 (Wugui lieche) [Train sans chemin]. Shanghai: Xiandai Chubanshe.

Lu, Xun (1930/1956): 硬譯與文學的階級性 («Yingyi» yu wenxue de jiejixing) [《La traduction raide» et la classe de la littérature]. In: Lu Xun quanji [CEuvres complètes de Lu Xun]. Beijing: Renmin wenxue chubanshe, 155-173.

Munang, Bao'er [Morand] (1922/1928): 六日之夜 (Liuri zhiye) [La nuit des six-jours]. In: 法蘭 西短篇傑作集(第一集) (Falanxi duanpian jiezuoji: di yi ji) [Chef-d'œuvre des nouvelles françaises], (traduit par Wangshu DAI). Vol.1. Shanghai: Shuimo chubanshe, 1-25.

Muhang, Bao'er [Morand] (1929): 天女玉麗 (Tiannu yuli) [Céleste Julie], (traduit par Wangshu DAI). Shanghai: Shanghai Shangzhi Shuwu.

NAKAMURA, Kan'ichi (1925/1980) : 新感覚派及びモオランの『夜開く』に就て (Shinkankakuha oyobi mōran no Yoru hiraku ni tsute) [Ả propos de l'École des sensations nouvelles et Ouvert 
la nuit de Morand]. In: Nihon bungaku kenkyū shiryō kankō-kai éd.: 横光利一と新感覚 派 (Yokomitsu Riichi to shinkankakuha) [Yokomitsu Riichi et l'École des sensations nouvelles]. Tokyo: Yūseidō, 227-238.

Òmura, Azusa (2011): The Paradox of Modernism: Paul Morand and the Literature of Japanese Modernism. Thèse de doctorat. Tokyo: Industrial University of Tokyo.

Tsuchiya, Satoshi (2006): 大正期における堀口大学の翻訳 (Taishō ki ni okeru Horiguchi Daigaku no hon'yaku) [La traduction d'Horiguchi Daigaku dans l'ère Taishō]. In: Nihon kindai bungaku-kai henshū iinkai, dir.: Nihon kindai bungaku [La littérature moderne japonaise]. $75: 87-102$.

Shimazaki, Tōson (1985): 島崎藤村全集 (Shimazaki Tōson zenshū) [Euvres complètes de Shimazaki Tōson]. Vol. 11. Tokyo: Chikuma shobō.

Starrs, Roy, dir. (2012): Rethinking Japanese Modernism. Leiden et Boston: Global Oriental.

Tayama, Katai (1977): 田山花袋集 (Tayama Katai shū) [Collection de Tayama Katai]. Tokyo: Chikuma shobo.

Thibault, Bruno (1992): L'Allure de Morand: du modernisme au pétainisme. Birmingham: Summa Publications.

WatANABE, Kazutami (1995): フランスの誘惑: 近代日本精神史史論 (Furansu no yūwaku: Kindai Nihon seishin-shi shiron) [La séduction de la France: essai sur l'histoire d'intellectuel japonais modern]. Tokyo: Iwanami shoten.

YAN, Jiayan: 前言 (Qianyan) [Introduction]. In: Jiayan YAN, dir. (1985): 新感覺派小說選 (Xinganjuepai xiaoshuo xuan) [Sélection de l'École des sensations nouvelles]. Beijing: Renmin wenxue chubanshe, 1-38.

ZHu, Qiaosen éd. (1996): 朱自清全集 (Zhu Ziqing quanji) [Euvres complètes de Zhu Ziqing]. Vol. 8. Nanjing: Jiangsu jiaoyu chubanshe. 